\title{
Constraining flavoured contact interactions at the LHC
}

\author{
Sacha Davidson ${ }^{a}$ and Sébastien Descotes-Genon ${ }^{b}$ \\ ${ }^{a} I P N L$, Université de Lyon, Université Lyon 1, CNRS/IN2P3, \\ 4 rue E. Fermi 69622, Villeurbanne cedex, France \\ ${ }^{b}$ Laboratoire de Physique Théorique, CNRS/Univ. Paris-Sud 11 (UM8627), \\ 91405 Orsay Cedex, France \\ E-mail: s.davidson@ipnl.in2p3.fr, descotes@th.u-psud.fr
}

ABSTRACT: Contact interactions are the low-energy footprints of New Physics, so ideally, constraints upon them should be as generic and model independent as possible. Hadron colliders search for four-quark contact interactions with incident valence quarks, and the LHC currently sets limits on a flavour sum (over $u u, d d$ and $u d$ ) of selected interactions. We approximately translate these bounds to a more complete (and larger) set of dimension-six interactions of specified flavours. These estimates are obtained at the parton level, are mostly analytic and are less restrictive than the experimental bounds on flavour-summed interactions. The estimates may scale in a simple way to higher energy and luminosity.

KEYwords: Hadronic Colliders

ArXIV EPRINT: 1311.5981 


\section{Contents}

1 Introduction 1

2 Kinematics of $p p \rightarrow$ dijets $\quad 2$

3 Contact interactions: operators versus effective interactions 4

3.1 Operators 4

3.2 Effective interactions 5

$\begin{array}{lll}3.3 & \text { Feynman rules and dimensional analysis } & 7\end{array}$

4 Contact interactions: flavour structure $\quad 8$

4.1 Impact of flavour on the search for contact interactions 8

$\begin{array}{ll}4.2 \text { Comparing to the existing notation } & 9\end{array}$

5 Estimating bounds on flavoured contact interactions $\quad 10$

$\begin{array}{lll}5.1 & \text { From data to partonic cross sections } & 10\end{array}$

$\begin{array}{lll}5.2 & \text { Bounds on flavoured operators } & 13\end{array}$

$\begin{array}{lll}5.3 & \text { Extrapolating to higher energy or luminosity } & 14\end{array}$

$\begin{array}{llr}6 & \text { Discussion } & 20\end{array}$

$\begin{array}{lll}7 & \text { Summary } & 21\end{array}$

A Kinematics and cross sections $\quad 22$

A.1 Definitions 23

A.2 $q_{i} g \rightarrow q_{i} g-$ QCD only 23

A.3 $q_{i} q_{j} \rightarrow q_{m} q_{n}$

A.3.1 $q q \rightarrow q q,(i=j=m=n) \quad 23$

A.3.2 $q q^{\prime} \rightarrow q q^{\prime}, i=m \neq j=n \quad 24$

A.3.3 $q^{\prime} q^{\prime} \rightarrow q q \quad 25$

A.3.4 $q^{\prime} q^{\prime \prime} \rightarrow q q$, or $q q \rightarrow q^{\prime} q^{\prime \prime} \quad 25$

A.3.5 $q^{\prime \prime} q \rightarrow q q^{\prime}$ and any vertex with more than three different flavours 26

A.4 $q_{i} \bar{q}_{m} \rightarrow \bar{q}_{j} q_{n} \quad 26$

\section{Introduction}

Contact interactions inevitably arise as the low-energy remnants of high-energy theories. Should the LHC not find (additional) new particles, it can nonetheless be sensitive to their traces in contact interactions.

This paper focuses on four-quark contact interactions, with two incoming valence quarks, as is most probable at the LHC. New Physics from beyond the LHC energy can induce various operators $[1,2]$, with different Lorentz and gauge structures as well as 
flavour indices. So bounds on contact interactions involving specific flavours are appropriate for constraining New Physics models. The great variety of high-energy models have different low-energy footprints, such as different flavour structures for contact interactions. For instance, the contact interactions involving singlet (i.e., right-handed) $u$ quarks might differ from those involving $d$ quarks. Furthermore, the flavour structure of contact interactions induced by New Physics is explored by precision flavour physics (mostly for flavour off-diagonal operators), so the collider bounds should also be for specific flavour indices, to allow comparison and combination with low-energy observations.

However, the current LHC bounds are given for a subset of flavour-summed operators [3-5]. The aim of this paper is to make an approximate translation of the collider constraints onto individual, flavoured operators and to illustrate the limits of such a translation. Two issues arise in attempting to apply the published contact interactions bounds to a different operator: the Lorentz and gauge structure of the operator affects the partonic cross section, and the flavour indices affect possible interferences with QCD, as well as controlling the probability of finding the initial state quarks in the proton. Let us mention that four-quark contact interactions have been searched for at the Tevatron in $q \bar{q} \rightarrow$ dijets [6], and at the LHC in $q q \rightarrow$ dijets [3-5]. In both cases, the initial state $q$ or $\bar{q}$ are first generation valence quarks, whereas the final state can be of any flavour, so the Tevatron and LHC can constrain different flavour structures. In this paper, we focus on the $q q \rightarrow$ dijets process at the LHC. Some previous theoretical analyses that use LHC dijet searches to constrain New Physics can be found in reference [7-11].

Section 2 reviews the kinematical variables used by the experimental collaborations [3, $4,6]$ to constrain contact interactions from the rapidity distribution of high energy dijets. These variables allow an approximate "factorisation" of the $p p \rightarrow$ dijets cross section into an integral over parton distribution functions (pdfs), multiplying a partonic cross section. In section 3.1, a basis of Standard Model gauge-invariant, dimension-six effective operators are listed, which, in the presence of electroweak symmetry breaking, induce the effective interactions listed in section 3.2. We will constrain the coefficients of these effective interactions, because, as discussed in section 3 , this allows to set conservative and operator-basis-independent limits, while turning the interactions on one at a time. Issues regarding flavour and interferences are discussed in section 4. Section 5 uses the approximate factorisation of section 2 to estimate bounds on individual operators based on the CMS analysis of ref. [4]. The appendix collects the partonic cross sections for various contact interactions. Its aim is to allow an interested reader to estimate bounds from future data on their selection of contact interactions, following the mostly analytic recipe given in section 5 .

\section{Kinematics of $p p \rightarrow$ dijets}

At the LHC, the cross section for $p p \rightarrow$ dijets contains contributions from QCD, electroweak bosons, and possibly from four-parton contact interactions. The purely QCD (or QED) contribution falls off as $1 / \hat{s}$, where $\hat{s}=M_{\text {dijet }}^{2}$ is the four-momentum-squared of the pair of jets, and grows in the forward/backward directions. On the other hand, the contact inter- 

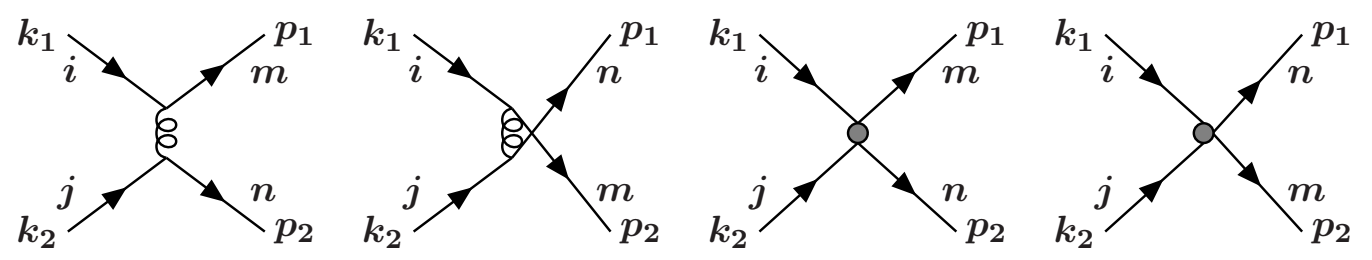

Figure 1. Possible QCD and contact interaction diagrams for $q_{i} q_{j} \rightarrow q_{m} q_{n}$. QED diagrams with the gluon replaced by a photon are also possible. At the grey blob representing the contact interaction, the quark lines may have chiral projectors and/or a colour matrix. Which gauge diagrams interfere with a given contact interaction will depend on its flavour indices and operator structure; for instance, for $V-A$ operators with $i=j=n=m$, all four gauge diagrams could interfere.

action contribution grows with $\hat{s}$ and is fairly central. So bounds on contact interactions can be obtained from the meagre population of central high energy dijets. With a clever choice of variables, the distribution in rapidity and invariant-mass squared of the two jets can be approximated as the partonic cross section, multiplying an integral of pdfs. We review here this approximation.

The parton-level diagrams for quark-quark scattering $q_{i}\left(k_{1}\right) q_{j}\left(k_{2}\right) \rightarrow q_{m}\left(p_{1}\right) q_{n}\left(p_{2}\right)$ are given in figure 1 , where $i, j, m, n$ are flavour indices, and $\{k, p\}$ are four-momenta. The resulting cross sections, which can be parametrised with the partonic mandelstam variables $\hat{s}, \hat{t}$, and $\hat{u}$ are listed in the appendix. To obtain an observable, the partons must be embedded in the incident protons, here taken to have four-momenta $P_{ \pm}$. We denote $f_{j}\left(x_{1}\right)$ the probability density that the parton $j$ carries a fraction $x_{1}$ of the four-momentum of an incident proton, so that $\hat{s}=x_{1} x_{2}\left(P_{+}+P_{-}\right)^{2}$. The total cross section can be written

$$
\sigma(p p \rightarrow \text { dijets })=\sum_{i, j, m, n} \int_{0}^{1} d x_{1} \int_{0}^{1} d x_{2} f_{i}\left(x_{1}\right) f_{j}\left(x_{2}\right) \sigma\left(i\left(x_{1} P_{+}\right) j\left(x_{2} P_{-}\right) \rightarrow m n\right)
$$

where the sum runs over all possible incident partons for each partonic process, and over all the partonic processes which contribute (e.g., in the case of dijets, $q q \rightarrow q q, q \bar{q} \rightarrow q \bar{q}, g g \rightarrow$ $q \bar{q}, g g \rightarrow g g$, etc). In our estimates, we only include $u u \rightarrow u u, d d \rightarrow d d, u d \rightarrow u d$ and $u g \rightarrow u g$ in the partonic QCD cross section. These should be the main contributions to the dijet cross section. The partonic cross sections increase by a factor $\sim 9 / 4$ for each initial gluon: $\sigma(q q \rightarrow q q): \sigma(q g \rightarrow q g): \sigma(g g \rightarrow g g) \sim \frac{4}{9}: 1: \frac{9}{4}$. However, the density of gluons in the proton, at the large values of $x$ which are relevant here, is at least a factor of $1 / 10(1 / 3)$ below that of valence $u(d)$ quarks, and the density of sea quarks, is two orders of magnitude below the $u$ density, which justifies our approximation.

It can be convenient to introduce the pseudo-rapidities

$$
y=\frac{1}{2} \ln \frac{E+p_{z}}{E-p_{z}}
$$

of the individual jets, and their combined mass squared

$$
M_{\text {dijet }}^{2}=\hat{s}=x_{1} x_{2} s .
$$

We interchangeably refer to $M_{\text {dijet }}^{2}$ or $\hat{s}$ throughout the paper, using the convention that partonic variables, such as the Mandelstam $\hat{s}, \hat{t}, \hat{u}$ (defined in eq. (A.1)), wear hats. 
The CMS search [4] for contact interactions in the angular distribution of dijets at high $M_{\text {dijet }}^{2}$ uses as variables the dijet mass squared eq. (2.3), the pseudo-rapidity of the partonic centre of mass frame

$$
y_{+}=\left(y_{1}+y_{2}\right) / 2
$$

and

$$
\chi=\exp \left|y_{1}-y_{2}\right|=\frac{1+\left|\cos \theta^{*}\right|}{1-\left|\cos \theta^{*}\right|},
$$

where $\theta^{*}$ is the centre-of-mass scattering angle away from the beam axis (see the appendix).

At the parton level, the QCD contributions to the differential cross section $d \hat{\sigma} / d \hat{t}$ have a $1 / \hat{t}^{2}$ divergence, leading to a large rate for small-angle scatterings along the beam pipe. On the other hand, the dijets produced by contact interactions have a more isotropic distribution. The most sensitive place to look for contact interactions is therefore in largeangle scatterings, producing dijets in the central part of the detector. Expressed as a function of $\chi$, the QCD contribution to the dijet cross section is approximately flat, whereas the contact interaction contribution peaks at small $\chi$. This is illustrated in figures 1 and 2 of ref. [4], where the main effect of contact interaction occurs for $1 \leq \chi \leq 3$ bin, which corresponds to $60^{\circ} \leq \theta^{*} \leq 120^{\circ}$.

With these variables, the differential dijet cross section is

$$
\frac{d \sigma}{d y_{+} d \chi d M_{\mathrm{dijet}}^{2}}=\frac{f_{i}\left(x_{1}\right) f_{j}\left(x_{2}\right)}{s} \frac{d \hat{\sigma}}{d \hat{t}} \frac{\hat{t}^{2}}{\hat{s}} .
$$

For fixed dijet mass, the pdfs depend on $y_{+}$but not $\chi$ (since $x_{1}=e^{y_{+}} M_{\text {dijet }} / \sqrt{s}, x_{2}=$ $e^{-y_{+}} M_{\text {dijet }} / \sqrt{s}$ ), and the partonic differential cross section depends on $\chi$ but not $y_{+}$, so the expected number of events can be factorised as a integral-of-pdfs, multiplied by a partonic cross section. Therefore, in an ideal world with contact interactions, the dijet distribution in mass and $\chi$ could allow one to determine the actual partonic cross section, providing the necessary information to identify the operator(s) that induced it.

\section{Contact interactions: operators versus effective interactions}

\subsection{Operators}

New Physics from a scale $M>m_{W}$ can be described, at scales $\ll M$, by an effective Lagrangian containing the renormalisable Standard Model (SM) interactions, and various $\mathrm{SU}(3) \times \mathrm{SU}(2) \times \mathrm{U}(1)_{Y}$-invariant operators of dimension $>4$, with coefficients determined by the New Physics model. There can be relations among the operators, arising from symmetries and equations of motion. This section gives a basis of dimension six, fourquark operators, taken from the Buchmuller-Wyler [1] list as pruned by ref. [2]. ${ }^{1}$

In the following list of four quark operators, $Q$ are electroweak doublets, $U$ and $D$ are singlets, $\lambda^{A}$ are the generators of $\mathrm{SU}(3), \vec{\tau} / 2$ are those of $\mathrm{SU}(2)$, the gauge index sums are implicit inside the parentheses, and $i, j, m, n$ are generation indices which all run from

\footnotetext{
${ }^{1}$ We restrict our analysis to dimension- 6 operators. This is a reasonable perturbative approximation when the next-order terms, relatively suppressed by $\sim \hat{s} / \Lambda^{2}, v^{2} / \Lambda^{2}$ can be neglected.
} 
$1 \ldots 3$ (so there is a factor $1 / 2$ in front of operators made of same current twice, to obtain the Feynman rule described below in eq. (3.17)).

$$
\begin{aligned}
\mathcal{O}_{\bar{Q} Q}^{1,1} & =\frac{1}{2}\left(\bar{Q}_{m} \gamma^{\mu} Q_{i}\right)\left(\bar{Q}_{n} \gamma_{\mu} Q_{j}\right) \\
\mathcal{O}_{\bar{Q} Q}^{1,3} & =\frac{1}{2}\left(\bar{Q}_{m} \gamma^{\mu} \vec{\tau} Q_{i}\right)\left(\bar{Q}_{n} \gamma_{\mu} \vec{\tau} Q_{j}\right) \\
\mathcal{O}_{\bar{U} U}^{1,1} & =\frac{1}{2}\left(\bar{U}_{m} \gamma^{\mu} U_{i}\right)\left(\bar{U}_{n} \gamma_{\mu} U_{j}\right) \\
\mathcal{O}_{\bar{D} D}^{1,1} & =\frac{1}{2}\left(\bar{D}_{m} \gamma^{\mu} D_{i}\right)\left(\bar{D}_{n} \gamma_{\mu} D_{j}\right)
\end{aligned}
$$

There are also operators contracting currents of singlet quarks of different charge:

$$
\mathcal{O}_{\bar{U} D}^{1,1}=\left(\bar{U}_{m} \gamma^{\mu} U_{i}\right)\left(\bar{D}_{n} \gamma_{\mu} D_{j}\right) \quad \mathcal{O}_{\bar{U} D}^{8,1}=\left(\bar{U}_{m} \gamma^{\mu} \lambda^{A} U_{i}\right)\left(\bar{D}_{n} \gamma_{\mu} \lambda^{A} D_{j}\right)
$$

and operators contracting doublet and singlet currents:

$$
\begin{array}{ll}
\mathcal{O}_{\bar{Q} D}^{1,1}=\left(\bar{Q}_{m} \gamma^{\mu} Q_{i}\right)\left(\bar{D}_{n} \gamma_{\mu} D_{j}\right) & \mathcal{O}_{\bar{Q} D}^{8,1}=\left(\bar{Q}_{m} \gamma^{\mu} \lambda^{A} Q_{i}\right)\left(\bar{D}_{n} \gamma_{\mu} \lambda^{A} D_{j}\right) \\
\mathcal{O}_{\bar{Q} U}^{1,1}=\left(\bar{Q}_{m} \gamma^{\mu} Q_{i}\right)\left(\bar{U}_{n} \gamma_{\mu} U_{j}\right) & \mathcal{O}_{\bar{Q} U}^{8,1}=\left(\bar{Q}_{m} \gamma^{\mu} \lambda^{A} Q_{i}\right)\left(\bar{U}_{n} \gamma_{\mu} \lambda^{A} U_{j}\right)
\end{array}
$$

and finally there is a scalar operator, with antisymmetric $\mathrm{SU}(2)$ index contraction across the parentheses:

$$
\mathcal{O}_{\bar{Q}}^{S, 1,1}=\left(\bar{Q}_{m} U_{i}\right)\left(\bar{Q}_{n} D_{j}\right) \quad \mathcal{O}_{\bar{Q} \bar{Q}}^{S, 8,1}=\left(\bar{Q}_{m} \lambda^{A} U_{i}\right)\left(\bar{Q}_{n} \lambda^{A} D_{j}\right)
$$

\subsection{Effective interactions}

Once electroweak symmetry is broken, a particular operator induces one or several effective interactions among mass eigenstates. We aim at constraining these effective interactions, one at a time, through the LHC searches on contact interactions.

This leads us to make a distinction between (gauge-invariant) operators, and effective interactions (having distinct external legs). The aim of this distinction is to address a general problem with setting bounds on the coefficients of gauge-invariant operators [13]: such bounds may not transfer, in a simple way, from one operator basis to another. The selection of SM gauge-invariant operators made in section 3.1 is not unique, as expected for a choice of basis. A different list of operators might be more suited to describing some models because they capture the symmetries of the model in a more economical way. Ideally, the constraints on four-fermion operator coefficients should be transferable from one operator basis to another. However, this is not possible if these constraints are obtained by turning on one operator at a time, as will be done here. One difficulty is that an operator, such as the $\mathrm{SU}(2)$ triplet of eq. (3.3), induces several four-fermion interactions, so quoting a bound on the operator loses the correlation carrying the information about which interaction the bound arose from. In addition, if the operators can interfere among themselves (for instance, the singlet and triple operators of eqs. (3.1) and (3.3) can interfere), then the bound on a sum of operators could be less restrictive than the bounds on single operators. 
To circumvent the problem of "basis-dependent" bounds, ${ }^{2}$ we follow ref. [12], and set limits on the coefficients of "effective four-quark interactions", These effective interactions should be distinct, so that they do not interfere among each other, and should include all the interactions induced by the effective operators. Unfortunately, these two requirements are not quite compatible; there are two interactions which can interfere, but we neglect this effect.

We obtain the list by considering the $\mathrm{U}(1) \times \mathrm{SU}(3)$ invariant operators generated after electroweak symmetry breaking by the previous basis of SM gauge invariant operators (decomposing the $\mathrm{SU}(2)$ doublets $Q$ into their components). These interactions almost never interfere among themselves (the exceptions are eqs. (3.12) and (3.16)), which ensures that the prediction of a sum of interactions will be the sum of the predictions of the interactions taken separately. The possibilities are the following:

- the "neutral-current" left-left or right-right interactions, $X=L$ or $R$ :

$$
\begin{aligned}
& \mathcal{O}_{u_{m} u_{i} u_{n} u_{j}}^{1, X}=\frac{1}{2}\left(\bar{u}_{m} \gamma^{\mu} P_{X} u_{i}\right)\left(\bar{u}_{n} \gamma_{\mu} P_{X} u_{j}\right) \\
& \mathcal{O}_{d_{m} d_{i} d_{n} d_{j}}^{1, X X}=\frac{1}{2}\left(\bar{d}_{m} \gamma^{\mu} P_{X} d_{i}\right)\left(\bar{d}_{n} \gamma_{\mu} P_{X} d_{j}\right) \\
& \mathcal{O}_{u_{m} u_{i} d_{n} d_{j}}^{1, X X}=\left(\bar{u}_{m} \gamma^{\mu} P_{X} u_{i}\right)\left(\bar{d}_{n} \gamma_{\mu} P_{X} d_{j}\right)
\end{aligned}
$$

Notice that, with the previous basis of gauge-invariant operators, the octet $\mathcal{O}_{\text {uudd }}^{8, R R}$ only arises for singlet currents.

- the "charged-current" interactions

$$
\mathcal{O}_{u_{m} d_{i} d_{n} u_{j}}^{1, C C}=\left(\bar{u}_{m} \gamma^{\mu} P_{L} d_{i}\right)\left(\bar{d}_{n} \gamma_{\mu} P_{L} u_{j}\right) \quad \mathcal{O}_{u_{m} d_{i} d_{n} u_{j}}^{8, C C}=\left(\bar{u}_{m} \gamma^{\mu} P_{L} \lambda^{A} d_{i}\right)\left(\bar{d}_{n} \gamma_{\mu} P_{L} \lambda^{A} u_{j}\right),
$$

the second of which can be rearranged to a linear combination of $\mathcal{O}_{u_{m} d_{i} d_{n} u_{j}}^{1, C C}$ and $\mathcal{O}_{u_{m} u_{j} d_{n} d_{i}}^{1, L L}$. We therefore do not include octet charged-current interactions. We include the singlet $\mathcal{O}_{u_{m} d_{i} d_{n} u_{j}}^{1, C C}$, which unfortunately can interfere with $\mathcal{O}_{u_{m} u_{j} d_{n} d_{i}}^{1, L L}$ (see eq. (A.14)).

- the "neutral-current" left-right operators,

$$
\begin{array}{ll}
\mathcal{O}_{u_{m} u_{i} u_{n} u_{j}}^{1, X}=\frac{1}{2}\left(\bar{u}_{m} \gamma^{\mu} P_{X} u_{i}\right)\left(\bar{u}_{n} \gamma_{\mu} P_{Y} u_{j}\right) & \mathcal{O}_{u_{m} u_{i} u_{n} u_{j}}^{8, X Y}=\frac{1}{2}\left(\bar{u}_{m} \gamma^{\mu} P_{X} \lambda^{A} u_{i}\right)\left(\bar{u}_{n} \gamma_{\mu} P_{Y} \lambda^{A} u_{j}\right) \\
\mathcal{O}_{d_{m} d_{i} d_{n} d_{j}}^{1, X Y}=\frac{1}{2}\left(\bar{d}_{m} \gamma^{\mu} P_{X} d_{i}\right)\left(\bar{d}_{n} \gamma_{\mu} P_{Y} d_{j}\right) & \mathcal{O}_{d_{m} d_{i} d_{n} d_{j}}^{8, X Y}=\frac{1}{2}\left(\bar{d}_{m} \gamma^{\mu} P_{X} \lambda^{A} d_{i}\right)\left(\bar{d}_{n} \gamma_{\mu} P_{Y} \lambda^{A} d_{j}\right) \\
\mathcal{O}_{u_{m} u_{i} d_{n} d_{j}}^{1, X Y}=\left(\bar{u}_{m} \gamma^{\mu} P_{X} u_{i}\right)\left(\bar{d}_{n} \gamma_{\mu} P_{Y} d_{j}\right) & \mathcal{O}_{u_{m} u_{i} d_{n} d_{j}}^{8, X Y}=\left(\bar{u}_{m} \gamma^{\mu} P_{X} \lambda^{A} u_{i}\right)\left(\bar{d}_{n} \gamma_{\mu} P_{Y} \lambda^{A} d_{j}\right)
\end{array}
$$

where $P_{X}, P_{Y} \in\left\{P_{L}, P_{R}\right\}, X \neq Y$.

\footnotetext{
${ }^{2}$ Ref. [14] shows some parameter choices where interference among operators could reduce sensitivity, although the $\mathcal{O}_{2}$ operator they discuss is not present in the basis of [2].
} 
- the scalar operators stemming from eq. (3.8), which each give two interactions:

$$
\begin{aligned}
\mathcal{O}_{q_{m} u_{i} q_{n} d_{j}}^{S 1} & =\left(\bar{d}_{m} P_{R} u_{i}\right)\left(\bar{u}_{n} P_{R} d_{j}\right)-\left(\bar{u}_{m} P_{R} u_{i}\right)\left(\bar{d}_{n} P_{R} d_{j}\right) \\
\mathcal{O}_{q_{m} u_{i} q_{n} d_{j}}^{S 8} & =\left(\bar{d}_{m} P_{R} \lambda^{A} u_{i}\right)\left(\bar{u}_{n} P_{R} \lambda^{A} d_{j}\right)-\left(\bar{u}_{m} P_{R} \lambda^{A} u_{i}\right)\left(\bar{d}_{n} P_{R} \lambda^{A} d_{j}\right) .
\end{aligned}
$$

For the scalar operators, we constrain the coefficients of the operators, that is, of $\mathcal{O}^{S 1}$ and $\mathcal{O}^{S 8}$ given above, because the interactions in the sums interfere between each other.

\subsection{Feynman rules and dimensional analysis}

Following the convention of collider constraints on contact interactions, we suppose that the coupling in the four-quark Feynman rule is

$$
i \eta \frac{4 \pi}{\Lambda^{2}}, \quad \text { with } \eta= \pm 1 .
$$

The operators/interactions, of the previous sections are normalised such that their coefficient should be $\eta 4 \pi / \Lambda^{2}$. For each flavoured contact interaction, there will be a different lower bound on $\Lambda$.

In the matrix element squared, the contact interaction may interfere with QCD (depending on its colour, flavour and chiral structure) as well as with itself. The $p p \rightarrow$ dijet cross section, in the central part of the detector, is therefore of the form

$$
\frac{d \sigma}{d \chi} \sim C_{\mathrm{QCD}} \frac{\alpha_{s}^{2}}{\hat{s}}+C_{2} \frac{\alpha_{s}}{\Lambda^{2}}+C_{4} \frac{\hat{s}}{\Lambda^{4}},
$$

where the $C_{x}$ are $\mathcal{O}(1)$ constants that depend on the specific contact interaction. $C_{4}$, related to the contribution of contact interaction alone, is always positive and will dominate for very large $\hat{s}$, whereas $C_{2}$, stemming from the interference between QCD and contact interactions, can be positive or negative depending on the value of $\eta$, and can thus induce either a deficit or an excess of events at intermediate values of $\hat{s}$.

Requiring that the contact interactions induce a deviation of $\epsilon \lesssim 1$ from the QCD expectation, gives

$$
\frac{1}{\Lambda^{2}} \lesssim \frac{\alpha_{s}}{\hat{s}},
$$

suggesting that for $\sqrt{\hat{s}}=3 \mathrm{TeV}$ at the $7-8 \mathrm{TeV}$ LHC [4], the limits on $\Lambda$ should be $\gtrsim$ $O(10) \mathrm{TeV}$. In the following, we will impose a tighter constraint on eq. (3.18) for specific contact interactions, providing different bounds depending on their flavour structure.

Similar estimates can be made for dimension-eight four quark operators, with coefficients $4 \pi \hat{s} / \Lambda_{8}^{4}$, or $4 \pi v^{2} / \Lambda_{8}^{4}$, where $v$ is the "v.e.v" of the Higgs field. Focussing on the former, because $v^{2} \ll M_{\text {dijet }}^{2}$, one obtains

$$
\frac{d \sigma}{d \chi} \sim C_{\mathrm{QCD}} \frac{\alpha_{s}^{2}}{\hat{s}}+C_{4}^{\prime} \frac{\alpha_{s} \hat{s}}{\Lambda_{8}^{4}}+C_{8} \frac{\hat{s}^{3}}{\Lambda_{8}^{8}}
$$

which can be interpreted in two ways. First, if there is New Physics at a scale $M^{2} \gtrsim \hat{s}$ which induces observable dimension six operators with coefficients $4 \pi / \Lambda_{6}^{2}$, then comparing 
the last term of eq. (3.18) to the middle term of eq. (3.20), with $C_{4} \simeq C_{4}^{\prime}$, shows that the contribution of dimension eight operators is relatively suppressed by $\alpha_{s} \Lambda_{6}^{4} / \Lambda_{8}^{4}$ [22]. Alternatively, if the coefficients of dimension 6 operators are suppressed, $\Lambda_{6} \gg \Lambda_{8}$, then colliders could still be sensitive to the narrow window $\hat{s}<\Lambda_{8}^{2}<\hat{s} / \sqrt{\alpha_{s}}$, via dimension eight operators.

The perturbative exchange of a boson of mass $M$, momentum $p$, and coupling $g^{\prime}$ to quarks is represented by an amplitude

$$
\mathcal{A} \sim \frac{g^{\prime 2}}{p^{2}-M^{2} \pm i M \Gamma} \rightarrow \frac{g^{\prime 2}}{M^{2}} \equiv \frac{4 \pi}{\Lambda^{2}} \lesssim \frac{g_{s}^{2}}{\hat{s}}
$$

where $p^{2}$ can be $\hat{s}, \hat{t}$ or $\hat{u}$. For $p^{2} \ll M^{2}$, this gives the contact interaction after the arrow, where also the estimated bound of (3.19) is included. Since this applies in the limit $M^{2} \gg \hat{s}$, there is almost no $\left\{g^{\prime}, M\right\}$ parameter space where both the limit is satisfied, and colliders have sensitivity. So it is interesting to consider $M^{2} \sim \hat{s}$. Equation (3.21) suggests that such a boson can contribute a contact-like interaction in the $t$ and $u$ channels (recall $t, u \leq 0$ ), or in the $s$-channel for a broad boson with $\Gamma \sim M$. So the common lore is that collider contact interaction searches can be sensitive to bosons with

$$
M \gtrsim M_{\text {dijet }}, g^{\prime} \gtrsim g_{s} \frac{M}{M_{\text {dijet }}} .
$$

\section{Contact interactions: flavour structure}

\subsection{Impact of flavour on the search for contact interactions}

Each of the interactions given in the section 3.2 exists in a plethora of flavoured combinations, only some of which can be constrained by colliders. The flavour indices have two effects on the collider bounds: first, some flavours are more plentiful in the proton than others, and second, the cross section can involve interferences with QCD or QED, which depend on the flavours.

First, at the LHC, bounds can be set on contact interactions with two incoming valence quarks $(u u, d d, u d)$. This is because the density of sea quarks and antiquarks is very suppressed. The incident partons should both carry a significant fraction of the proton momentum, to produce a pair of jets of large combined mass (the last bin in dijet mass of the CMS analysis [4] is $M_{\text {dijet }}>3 \mathrm{TeV}$ at the $7 \mathrm{TeV}$ LHC, corresponding to $x_{1} x_{2} \geq 9 / 49$ ). At such large values of $x$, the density of all flavours of sea quark and anti-quark is of similar size and about two orders of magnitude below the density of $u$ quarks. It is therefore doubtful, with current data, to set a bound on contact interactions with an initial sea quark or anti-quark, because a huge cross section would be required to compensate the relative suppression of sea pdfs.

The second effect of flavour indices is on the partonic cross section. For instance, the cross section for $u d \rightarrow u d$, mediated by QCD + contact interactions, is different from $u u \rightarrow u u$ (see eqs. (A.4)-(A.9)). In particular, the singlet interactions mediating $u u \rightarrow u u$ interfere with QCD, whereas there is not interference between QCD and singlet interactions mediating $u d \rightarrow u d$. Flavour-summed contact interactions constrained by the experimental collaborations have thus a reduced sensitivity to destructive interferences as they get 


\begin{tabular}{|c|c|c|}
\hline & $\left(\eta_{L L}, \eta_{R R}, \eta_{L R}\right)$ & operators \\
\hline$\Lambda_{L L}^{ \pm}$ & $( \pm 1,0,0)$ & $\pm\left[\mathcal{O}_{\text {uuuu }}^{1, L L}+2 \mathcal{O}_{\text {uudd }}^{1, L L}+\mathcal{O}_{d d d d}^{1, L L}\right]$ \\
$\Lambda_{R R}^{ \pm}$ & $(0, \pm 1,0)$ & $\pm\left[\mathcal{O}_{\text {uuuu }}^{1, R R}+2 \mathcal{O}_{\text {uudd }}^{1, R R}+\mathcal{O}_{d d d d}^{1, R R}\right]$ \\
$\Lambda_{V V}^{ \pm}$ & $( \pm 1, \pm 1, \pm 1)$ & $\pm\left[\sum_{m, n=u, d}\left(\mathcal{O}_{m m n n}^{1, L L}+\mathcal{O}_{m m n n}^{1, L R}+\mathcal{O}_{m m n n}^{1, R L}+\mathcal{O}_{m m n n}^{1, R R}\right)\right]$ \\
$\Lambda_{A A}^{ \pm}$ & $( \pm 1, \pm 1, \mp 1)$ & $\pm\left[\sum_{m, n=u, d}\left(\mathcal{O}_{m m n n}^{1, L L}-\mathcal{O}_{m m n n}^{1, L R}-\mathcal{O}_{m m n n}^{1, R L}+\mathcal{O}_{m m n n}^{1, R R}\right)\right]$ \\
$\Lambda_{V-A}^{ \pm}$ & $(0,0, \pm 1)$ & $\pm\left[\mathcal{O}_{\text {uuuu }}^{1, L R=R L}+\mathcal{O}_{\text {uudd }}^{1, L R}+\mathcal{O}_{d d u u}^{1, L R}+\mathcal{O}_{d d d d}^{1, L R=R L}\right]$ \\
\hline
\end{tabular}

Table 1. Sums of operators contributing (at the LHC) to some commonly studied contact interactions (see eq. (4.1)). On the left, the sub/super-scripts for $\Lambda$ indicate the choice of $\eta$ coefficients. On the right are given the corresponding interactions from section 3.2. In principle, contact interactions studied in collider experiments have a sum over all flavours; however, the LHC is principally sensitive to contact interactions with two incoming valence quarks, so the flavour sum is over $u, d$.

contributions from several flavour operators, most of them being positive contributions coming from contact interactions alone that increase the cross sections. In the appendix, we attempt to classify the possible flavour index combinations, and provide their partonic cross sections.

Another comment is in order at this stage. The experimental final state considered here is a pair of jets, so the final state quarks can be of any flavour other than top. This means the LHC is sensitive to curious $\Delta F=2$ and $\Delta F=1$ flavour-changing operators mediating processes like $u u \rightarrow c c$ or $u d \rightarrow u b$. Flavour physics (e.g. meson mixing and $B$ decays) can impose more stringent bounds on some of them. In the following, we will give the LHC bounds on the various flavour-changing operators.

\subsection{Comparing to the existing notation}

Traditionally [15], collider searches for contact interactions quote bounds on the mass scale $\Lambda$ appearing in an interaction of the form:

$$
\begin{gathered}
\mathcal{L}_{\text {Pythia }}=\frac{4 \pi}{\Lambda^{2}} \sum_{i, j=u, d, s, c, b}\left[\frac{\eta_{L L}}{2}\left(\bar{q}_{i} \gamma^{\mu} P_{L} q_{i}\right)\left(\bar{q}_{j} \gamma_{\mu} P_{L} q_{j}\right)+\frac{\eta_{R R}}{2}\left(\bar{q}_{i} \gamma^{\mu} P_{R} q_{i}\right)\left(\bar{q}_{j} \gamma_{\mu} P_{R} q_{j}\right)\right. \\
\left.+\eta_{L R}\left(\bar{q}_{i} \gamma^{\mu} P_{R} q_{i}\right)\left(\bar{q}_{j} \gamma_{\mu} P_{L} q_{j}\right)\right] .
\end{gathered}
$$

Specifically, this is the contact interaction coded into PYTHIA $8[16,17]$, where the $\eta$ coefficients can be chosen to be \pm 1 or 0 . Some frequently studied combinations of $\eta$ coefficients are given in table 1.

With the aim of obtaining useful and conservative bounds, our constraints will differ in three ways:

1. We include the octet operators $\mathcal{O}^{8}$ of section 3.2. They generally give smaller modifications to the dijet rate, so the bound on $\Lambda$ is lower, as can be seen from the tables of section 5 . 
2. We constrain each combination of flavour indices separately.

3. We only consider $\mathcal{O}^{1, L R}$ (or $\mathcal{O}^{1, R L}$ ), and $\mathcal{O}^{1, L L}$ (or $\mathcal{O}^{1, R R}$ ), but not the various linear combinations available in table 1.

Turning on one effective interaction of given flavours at a time (as done here) gives conservative bounds if two conditions are satisfied. First, the interactions should not interfere among themselves, so that the contribution of the sum is the sum of the contributions. This is almost the case for the interactions of section 3.2. Second, each bound should arise from requiring that the operator not induce an excess of events (as opposed to a deficit of events). This will be true for the bounds that we will derive.

Suppose now that one wishes to set a bound on a specific New Physics model, which induces a sum of low-energy contact interactions - for instance the $V V$ combination. It is simple and conservative to take the strongest of the bounds obtained one-at-a-time for the operators in the sum. However, the true limit should be better. It is not straightforward to obtain the bound on $\Lambda_{V V}^{+}$given the limits on $\Lambda_{X X}^{+}(X=R$ or $L)$ and $\Lambda_{L R}^{+}$. Despite that the excess events induced by $\mathcal{O}^{1, V V}$ are the sum of the excesses due to $\mathcal{O}^{1, X Y}$ and $\mathcal{O}^{1, X X}$ (for $X \neq Y \in\{L, R\}$ ), there are two hurdles to obtaining a bound on $\Lambda_{V V}^{+}$: first, one must calculate the partonic cross section for the $V V$ operator combination, then one must know how it is constrained by the data. To address the first hurdle, we collect in the appendix the partonic cross sections for a variety of contact interactions. The second hurdle is a problem, because it is clear that the experimental collaborations cannot constrain all possible combinations of all contact interactions. However, contact interactions induce excess high-mass dijets in the central part of the detector, so observables which measure this, such as $F_{\chi}\left(M_{\text {dijet }}\right)[3]$ of the ATLAS collaboration, should be translatable to limits on generic contact interactions.

\section{Estimating bounds on flavoured contact interactions}

\subsection{From data to partonic cross sections}

Suppose that an effective interaction has been selected, with incident flavours of the first generation. The recipe to guess a bound on $\Lambda$, from the dijet distribution in $M_{\text {dijet }}^{2}$ and $\chi$, is simple:

1. look up the (flavoured) partonic cross section for the selected contact interaction plus $\mathrm{QCD}$, and evaluate at the $\chi$ corresponding to the bin.

2. integrate the pdfs of the incident partons over the $y_{+}$values which are consistent with the experimental cuts.

3. multiply 1) by 2), and require that it agree with the QCD expectation for the bin.

We want to illustrate in detail the procedure in the case of the CMS analysis [4]. The CMS collaboration measured the distribution of dijets in $\chi$ from $1 \rightarrow 16$, and $M_{\text {dijet }}$ from 0.4 to $\geq 3 \mathrm{TeV}$ [4]. We focus on the highest $M_{\text {dijet }}>3 \mathrm{TeV}$ bin (obtained with $2.2 \mathrm{fb}^{-1}$ of data), 
for which CMS plots the normalised ${ }^{3}$ differential cross sections $\left(\equiv \frac{1}{\sigma} \frac{d \sigma}{d \chi}\right)$, corresponding to the data, to the QCD expectation, and to the predictions of QCD plus contact interactions (denoted QCD+CI). As indicated in section 2, the highest sensitivity to contact interactions is obtained for the $1 \leq \chi \leq 3$ bin. A ratio which can be extracted for a specific bin is

$$
\frac{1}{\sigma_{\mathrm{QCD}+\mathrm{CI}}} \frac{d \sigma_{\mathrm{QCD}+\mathrm{CI}}}{d \chi}\left[\frac{1}{\sigma_{\mathrm{QCD}}} \frac{d \sigma_{\mathrm{QCD}}}{d \chi}\right]^{-1}
$$

where all the cross sections are for $p p \rightarrow$ dijets (partonic cross sections will wear hats):

$$
\begin{gathered}
\frac{d \sigma_{\mathrm{QCD}+\mathrm{CI}}}{d \chi}=\sum_{i, j, m, n} \int d y_{+} f_{i} f_{j}\left(\frac{d \hat{\sigma}_{\mathrm{QCD}}}{d \chi}(i j \rightarrow m n)+\frac{d \hat{\sigma}_{\mathrm{QCD} * \mathrm{CI}}}{d \chi}(i j \rightarrow m n)\right. \\
\left.+\frac{d \hat{\sigma}_{\mathrm{CI}}}{d \chi}(i j \rightarrow m n)\right)
\end{gathered}
$$

and are summed over the $\chi$ and $\hat{s}$ ranges of the bin (we will return to these sums later).

Clearly, the prediction of contact interactions should be compared with the data, not with the QCD prediction. However, we notice that the data [3, 4] agree with the QCD prediction (for $M_{\text {dijet }}>3 \mathrm{TeV}$ they are marginally below the predictions for low- $\chi$ bins and above for high- $\chi$ bins, in the case of ref. [4]). So we "normalise" our (incomplete) leading order parton-level QCD cross section, to the QCD expectation obtained by ATLAS and CMS at next-to-leading order (NLO) with hadronisation and detector effects. Then we estimate bounds on contact interactions by requiring that they add $\lesssim 1.6 \sigma$ to the QCD contribution, where $\sigma$ is the experimental statistical and systematic uncertainties for the relevant bin, added in quadrature. On the basis of the results of ref. [4], we estimate that this allows contact interactions to contribute from $1 / 3$ to $1 / 2$ of the QCD contribution either positively or negatively. In other words, even though we base our analysis on the most sensitive $\chi$-bin (from 1 to 3 ) at maximal $M_{\text {dijet }}$, which has an observed value slightly below the QCD prediction, we consider that the spread of data with respect to QCD in the other bins prevents us from interpreting the deficit in the 1-3 bin as a negative contribution from contact interactions. We thus take the more conservative approach to set a bound on contact interactions as a fraction (positive or negative) from QCD (we will come back to this point in section 5.3).

The ratio (5.1) can be related, in a series of steps, to a ratio of partonic cross sections.

- The first step consists in canceling $\sigma_{\mathrm{QCD}+\mathrm{CI}} \simeq \sigma_{\mathrm{QCD}}$ in the ratio. This is a selfconsistent approximation, because contact interactions only contribute in the low $\chi$ bins, where they are bounded to be a fraction of QCD.

- The second step amounts to writing the ratio

$$
\frac{d \sigma_{\mathrm{QCD}+\mathrm{CI}}}{d \chi}\left[\frac{d \sigma_{\mathrm{QCD}}}{d \chi}\right]^{-1}=1+\epsilon
$$

\footnotetext{
${ }^{3}$ At this stage, we suppress the $M_{\text {dijet }}^{2}$ dependence; $\frac{d \sigma}{d \chi}$ means $\int d y_{+} \frac{d^{3} \sigma}{d y_{+} d M_{\text {dijet }}^{2} d \chi}$, and $\sigma=$ $\int d \chi d y_{+} \frac{d^{3} \sigma}{d y_{+} d M_{\mathrm{dijet}}^{2} d \chi}$
} 
where

$$
\epsilon=\frac{\sum_{m, n} \int d \hat{s} \int d y_{+} f_{i} f_{j} \delta \frac{d \hat{\sigma}}{d \chi}\left(q_{i} q_{j} \rightarrow q_{m} q_{n}\right)}{\sum_{i, j, m, n} \int d \hat{s} \int d y_{+} f_{i} f_{j} \frac{d \hat{\sigma}_{Q C D}}{d \chi}\left(q_{i} q_{j} \rightarrow q_{m} q_{n}\right)},
$$

where the partonic differential cross section $\delta \frac{d \hat{\sigma}}{d \chi}$ is the modification to $\left(q_{i} q_{j} \rightarrow q_{m} q_{n}\right)$ induced by contact interactions (alone or through interference with QCD, corresponding to the last two terms inside the parentheses of eq. (5.2)). The denominator amounts to the QCD contribution which is summed over the possible initial flavour combinations (limited to $u u, d d, u d$ and $u g$ ). The integral over the $\hat{s}=M_{\text {dijet }}^{2}$ range of the bin has been reinstated.

- The third step corresponds to factorising the integrals in the ratio $1+\epsilon$. As discussed in section 2, the partonic cross sections depend on $M_{\text {dijet }}^{2}$ and $\chi$, and the pdfs depend on $M_{\text {dijet }}^{2}$ and $y_{+}$. We now want to factor the partonic cross section out of the integral across the $M_{\text {dijet }}>3 \mathrm{TeV}$ bin. This will be an acceptable approximation, because the pdfs drop rapidly with large increasing $x$, so contribute most of the integral in a narrow range of $M_{\text {dijet }} \sim 3 \mathrm{TeV}$.

We factorise in two steps, starting with the denominator of eq. (5.4). All the QCD cross sections scale as $1 / \hat{s}$, so the cross sections evaluated at $\hat{s}_{\min }=(3 \mathrm{TeV})^{2}$ can be factored out of the integrals and replaced by $\hat{s}_{\min } / \hat{s}$ as far as the $s$-dependence is concerned (see appendix). We are left to integrate for $N=-1$

$$
I_{i j}^{N}=\int_{\min }^{\max } d \hat{s}\left(\frac{\hat{s}}{\hat{s}_{\min }}\right)^{N} \int d y_{+} f_{i}\left(x_{1}\right) f_{j}\left(x_{2}\right), x_{1}=e^{y_{+}} \sqrt{\frac{\hat{s}}{s}}, x_{2}=e^{-y_{+}} \sqrt{\frac{\hat{s}}{s}},
$$

over the $y_{+}$region consistent with the CMS cuts $\left(y_{+}<1.1\right)$ and the value of $M_{\text {dijet }}^{2}$ (corresponding to $2 y_{+}<\ln s / \hat{s}$ ), and over the range of energy from $\hat{s}_{\min }=(3 \mathrm{TeV})^{2}$ to $\hat{s}_{\max }=(6 \mathrm{TeV})^{2}$. We use CTEQ10 [18] pdfs (at NLO) at a scale of $3 \mathrm{TeV}$. The results, normalised to $I_{u u}^{-1}$, are given in table 2 . The ratios in the table change by only a few percent when we change $\hat{s}_{\max }$ to $(4.5 \mathrm{TeV})^{2}$.

The second step is to take $\delta \frac{d \hat{\sigma}}{d \chi}$, evaluated at $\hat{s}=(3 \mathrm{TeV})^{2}$, out of the integral in the numerator of eq. (5.4). We have then to evaluate $I_{i j}^{N}$ for $N=1,0$, which corresponds to the $\hat{s}$ dependence of the $|\mathrm{CI}|^{2}$ and interference contributions respectively. For $i j=u u, d d$ or $u d$, these integrals can be up to $20 \%$ larger than for $N=-1$. We conservatively neglect this effect, and use the $r_{i j}$ given in table 2 .

In the end, as desired, we have obtained an analytic formulation of the experimental bound on contact interactions. The data gives $\epsilon \lesssim 1 / 3$ or $1 / 2$. For a contact interaction selected from section 3.2 , with incoming flavours $i j$,

$$
\epsilon=\frac{r_{i j} \delta \frac{d \hat{\sigma}}{d \chi}\left(q_{i} q_{j} \rightarrow q_{m} q_{n}\right)}{\left(1+r_{d d}\right) \frac{d \hat{\sigma}_{Q C D}}{d \chi}(u u \rightarrow u u)+r_{u d} \frac{d \hat{\sigma}_{Q C D}}{d \chi}(u d \rightarrow u d)+r_{u g} \frac{d \hat{\sigma}_{Q C D}}{d \chi}(u g \rightarrow u g)}
$$

where $r_{i j}$ is from table $2, \delta \frac{d \hat{\sigma}}{d \chi}$ is the parton-level excess with respect to QCD induced by contact interactions (given by the appendix), whereas the cross sections in the denominator are induced by QCD (see eqs. (A.4), (A.3) and (A.9)). 


\begin{tabular}{|c|c|}
\hline incoming partons $i j$ & $\begin{array}{c}r_{i j}=\text { integrated density } \\
\text { (normalised to } u u \text { ) }\end{array}$ \\
\hline$u u$ & 1 \\
$d d$ & 0.085 \\
$u d$ & 0.56 \\
$u g$ & 0.22 \\
\hline
\end{tabular}

Table 2. The ratios of integrated pdfs, $r_{i j}=I_{i j}^{-1} / I_{u u}^{-1}$, which allow translating the experimental bound on $\sigma(p p \rightarrow$ dijets $)$ to a bound on partonic cross sections. $r_{u d}$ and $r_{u g}$ are multiplied by 2 because the $u$ valence quark could come from either incoming proton.

\subsection{Bounds on flavoured operators}

We are now in a position to translate the CMS results in terms of flavoured operators. The recipe to guess a bound on $\Lambda$, as given in the previous section, can now be reformulated analytically:

1. look up in the appendix the contribution to the partonic cross section of the selected contact interaction(s) plus interference with QCD, and evaluate it at the $\chi$ corresponding to the bin of interest. ${ }^{4}$ The same must be done for the QCD cross sections (A.4), (A.3) and (A.9).

2. weight the various contact interactions by the appropriate $r_{i j}$ factor from table 2 , and the QCD cross sections as given in the denominator of eq. (5.6).

3. impose that the ratio of eq. (5.6), $\epsilon \leq 1 / 3$ (or $1 / 2$ ), which gives a quadratic polynomial for $\hat{s} / \Lambda^{2}$ whose root gives the estimated bound on $\Lambda$ for $\hat{s}$ taken at the lower end of the range allowed in the highest dijet mass bin.

In practice, we integrate the partonic cross sections over $1 \leq \chi \leq 3$, and plot $1+\epsilon$ in figures 2,3 and 4 . Imposing $\epsilon \leq 1 / 3$ or $1 / 2$ gives the bounds on $\Lambda$ in the tables 3 to 8 , stemming from the ratio $M_{\text {dijet }}^{2} / \Lambda^{2}$, by taking $M_{\text {dijet }}=3 \mathrm{TeV}[4]$.

The bound that we obtain on $\Lambda$ is the solution of a quadratic polynomial in $\hat{s} /\left(\alpha_{s} \Lambda^{2}\right)$ (see eq. (3.18)), so depends on the numerical value of $\alpha_{s}$. We take $\alpha_{s}(3 \mathrm{TeV}) \simeq 0.09$, in agreement with the leading order running, since we do a leading order calculation (this is analogous to using $\alpha_{s}\left(m_{Z}\right)=.139$ in PYтніA [19, 20]). If instead, we take the Particle Data Group value [21] $\alpha_{s}\left(m_{Z}\right) \simeq .12$, then $\alpha_{s}(3 \mathrm{TeV}) \simeq 0.08$. If the scale of evaluation of $\alpha_{s}$ is changed by a factor of two, $\alpha_{s}$ varies by about 0.005 . We conclude that varying $\alpha_{s}$ between 0.08 and 0.09 gives some notion of the NLO uncertainties, and take the larger value, since this yields the more conservative limit.

The study performed here neglects several effects, such as hadronisation (partons are not jets), and NLO corrections (calculated for $\mathcal{O}^{1, L L}$ in ref. [14]). We also do not consider dimension 8 operators: as pointed out by ref. [22], matrix elements to which QCD contributes could also have an $\mathcal{O}\left(1 / \Lambda^{4}\right)$ term from QCD interference with a dimension 8 operator. This interference is in principle suppressed with respect to dimension 6-contributions by a factor $\alpha_{s}$.

\footnotetext{
${ }^{4}$ For instance, $1 \leq \chi \leq 3$ corresponds to the range in $(-\hat{t},-\hat{u})$ between $\left(\frac{3}{4} \hat{s}, \frac{1}{4} \hat{s}\right)$ and $\left(\frac{1}{4} \hat{s}, \frac{3}{4} \hat{s}\right)$.
} 


\begin{tabular}{|l|c|cc|c|}
\hline Operator & flavours minj & $\hat{s} / \Lambda^{2}$ & $\Lambda<$ \\
\hline$+\mathcal{O}^{8, X Y}$ & $\bar{u} u \bar{u} u$ & .64 & .72 & $3.8-3.5 \mathrm{TeV}$ \\
$-\mathcal{O}^{8, X Y}$ & $\bar{u} u \bar{u} u$ & .20 & .28 & $6.7-5.7 \mathrm{TeV}$ \\
& & & & \\
$+\mathcal{O}^{1, X X}$ & $\bar{u} u \bar{u} u$ & .19 & .21 & $6.9-6.5 \mathrm{TeV}$ \\
$-\mathcal{O}^{1, X X}$ & $\bar{u} u \bar{u} u$ & .06 & .09 & $12.2-10 \mathrm{TeV}$ \\
$+\mathcal{O}^{1, X Y}$ & $\bar{u} u \bar{u} u$ & .19 & .23 & $6.9-6.3 \mathrm{TeV}$ \\
$-\mathcal{O}^{1, X Y}$ & $\bar{u} u \bar{u} u$ & .15 & .19 & $7.7-6.9 \mathrm{TeV}$ \\
\hline$+\mathcal{O}^{1, X X}$ & $\bar{d} d \bar{d} d$ & .43 & .52 & $4.6-4.2 \mathrm{TeV}$ \\
$-\mathcal{O}^{1, X X}$ & $\bar{d} d \bar{d} d$ & .31 & .39 & $5.4-4.8 \mathrm{TeV}$ \\
$+\mathcal{O}^{1, X Y}$ & $\bar{d} d \bar{d} d$ & .60 & .74 & $3.9-3.5 \mathrm{TeV}$ \\
$-\mathcal{O}^{1, X Y}$ & $\bar{d} d \bar{d} d$ & .56 & .70 & $4.0-3.6 \mathrm{TeV}$ \\
\hline$+\mathcal{O}^{\text {pythia }, X X}$ & & .15 & .17 & $7.7-7.3 \mathrm{TeV}$ \\
& & & & $(8.4 \mathrm{TeV})$ \\
$-\mathcal{O}^{\text {pythia, } X X}$ & & .06 & .07 & $12.2-11.3 \mathrm{TeV}$ \\
& & & & $(11.7 \mathrm{TeV})$ \\
$+\mathcal{O}^{\text {pythia, } X Y}$ & & .17 & .20 & $7.3-6.7 \mathrm{TeV}$ \\
$-\mathcal{O}^{\text {pythia, } X Y}$ & & & & $(8.0 \mathrm{TeV})$ \\
& & .13 & .17 & $8.3-7.3 \mathrm{TeV}$ \\
& & & & $(8.0 \mathrm{TeV})$ \\
\hline
\end{tabular}

Table 3. Estimated bounds on the contact interaction scale, obtained from figure 2. The interactions of the first column are from section 3.2 , and $\mathcal{O}^{\text {pythia }}$ is the flavour-summed operator of eq. (4.1) for comparison. The flavour indices of the second column are in the order of the fields in the operator, and correspond to $i j \rightarrow m n$. The bounds are for $\alpha_{s}\left(M_{\text {dijet }}\right)=0.09$. In the third column are given the bounds on $\hat{s} / \Lambda^{2}$ from requiring that the relative excess of dijets induced by contact interactions be $|\epsilon|<1 / 3$ or $|\epsilon|<1 / 2$. The bound in the last column is obtained with $\hat{s}=M_{\text {dijet }}^{2}=(3 \mathrm{TeV})^{2}$, for the two values of $\epsilon$. The bounds in parentheses on $\mathcal{O}^{\text {pythia }}$ are those of CMS [4].

We do include interference between QED and contact interactions, when there is no interference with QCD, but we neglect effects of weak interactions. There are (almost) no gluon contributions in this analysis: we include the $g q \rightarrow g q$ contribution to the QCD cross section, but neglect $g g \rightarrow g g$ (because we assume $f_{g} \lesssim f_{d} / 3 \lesssim f_{u} / 9$ ). We hope to consider $g g \bar{q} q$ contact interactions [23] in a later analysis.

\subsection{Extrapolating to higher energy or luminosity}

The reach of the future LHC for the usual flavour-summed contact interactions has been studied in ref. [24], who find expected limits $\Lambda \gtrsim 20 \mathrm{TeV}$. If the LHC with more energy and luminosity still does not find contact interactions, how would our flavoured estimates scale? 


\begin{tabular}{|c|c|cc|c|}
\hline Operator & flavours minj & $\hat{s} / \Lambda^{2}$ & $\Lambda<$ \\
\hline$+\mathcal{O}^{8, X X}$ & $\bar{d} d \bar{u} u$ & .59 & .67 & $3.9-3.7 \mathrm{TeV}$ \\
$-\mathcal{O}^{8, X X}$ & $\bar{d} d \bar{u} u$ & .21 & .28 & $6.4-5.7 \mathrm{TeV}$ \\
$-\mathcal{O}^{8, X Y}$ & $\bar{d} d \bar{u} u$ & .50 & .66 & $4.1-3.7 \mathrm{TeV}$ \\
$\mathcal{O}^{S 8}$ & $\bar{d} d \bar{u} u$ & .60 & .74 & $3.9-3.5 \mathrm{TeV}$ \\
& & & & \\
$+\mathcal{O}^{1, X X}$ & $\bar{d} d \bar{u} u$ & .17 & .22 & $7.1-6.4 \mathrm{TeV}$ \\
$-\mathcal{O}^{1, X X}$ & $\bar{d} d \bar{u} u$ & .16 & .20 & $7.3-6.7 \mathrm{TeV}$ \\
$+\mathcal{O}^{1, C C}$ & $\bar{u} d \bar{d} u$ & .27 & .31 & $5.8-5.4 \mathrm{TeV}$ \\
$-\mathcal{O}^{1, C C}$ & $\bar{u} d \bar{d} u$ & .10 & .14 & $9.5-8.0 \mathrm{TeV}$ \\
$+\mathcal{O}^{1, X Y}$ & $\bar{d} d \bar{u} u$ & .33 & .41 & $5.1-4.7 \mathrm{TeV}$ \\
$-\mathcal{O}^{1, X Y}$ & $\bar{d} d \bar{u} u$ & .31 & .39 & $5.2-4.8 \mathrm{TeV}$ \\
$\mathcal{O}^{S 1}$ & $\bar{d} d \bar{u} u$ & .39 & .48 & $4.8-4.3 \mathrm{TeV}$ \\
\hline
\end{tabular}

Table 4. Estimated bounds on the contact interaction scale, obtained from figure 3 for the interactions of the first column with $i j \rightarrow m n=u d \rightarrow u d$ flavour structure. In the third column are given the bounds on $\hat{s} / \Lambda^{2}$ from requiring $|\epsilon|<1 / 3$ or $|\epsilon|<1 / 2$. The last column is obtained with $\hat{s}=M_{\text {dijet }}^{2}=(3 \mathrm{TeV})^{2}$.

\begin{tabular}{|c|c|c|c|}
\hline Operator & flavours minj & $\hat{s} / \Lambda^{2}$ & $\Lambda<$ \\
\hline $\mathcal{O}^{8, X Y}$ & $\bar{c} u \bar{c} u$ & $.35 \quad .44$ & $5.1-4.5 \mathrm{TeV}$ \\
\hline $\mathcal{O}^{1, X X}$ & $\bar{c} u \bar{c} u$ & .11 .13 & $9.0-8.3 \mathrm{TeV}$ \\
\hline $\mathcal{O}^{1, X Y}$ & $\bar{c} u \bar{c} u$ & $.17 \quad .21$ & $7.3-6.5 \mathrm{TeV}$ \\
\hline $\mathcal{O}^{1, X X}$ & $\bar{s} d \bar{s} d, \bar{b} d \bar{b} d$ & $.37 \quad .45$ & $4.9-4.5 \mathrm{TeV}$ \\
\hline $\mathcal{O}^{1, X Y}$ & $\bar{s} d \bar{s} d, \bar{b} d \bar{b} d$ & $.59 \quad .71$ & $3.9-3.6 \mathrm{TeV}$ \\
\hline $\mathcal{O}^{1, X X}$ & $\bar{s} d \bar{b} d$ & $\begin{array}{ll}.30 & .37\end{array}$ & $5.5-4.9 \mathrm{TeV}$ \\
\hline $\mathcal{O}^{1, X Y}$ & $\bar{s} d \bar{b} d$ & $.59 \quad .71$ & $3.9-3.6 \mathrm{TeV}$ \\
\hline
\end{tabular}

Table 5. Estimated bounds on the contact interaction scale, obtained from figure 4, for the interactions of the first column with $i j \rightarrow m n$ flavour structure, $i j=u u$ or $d d$, and $\Delta F=2$. In the third column are given the bounds on $\hat{s} / \Lambda^{2}$ from requiring $|\epsilon|<1 / 3$ or $|\epsilon|<1 / 2$. The bound in the last column is obtained with $\hat{s}=M_{\text {dijet }}^{2}=(3 \mathrm{TeV})^{2}$.

The bounds obtained here are on the dimensionless variable $\hat{s} / \Lambda^{2}$, and they depend on the experimental uncertainty via $\epsilon$, as well as the scale at which the pdfs were evaluated. There are two useful approximations/assumptions:

1. suppose that the ratios of integrated pdfs, $r_{i j}$, given in table 2 , will not change significantly in going from the 8 to $14 \mathrm{TeV}$ LHC. 


\begin{tabular}{|c|c|c|c|}
\hline Operator & flavours $\operatorname{minj}$ & $\hat{s} / \Lambda^{2}$ & $\Lambda<$ \\
\hline $\mathcal{O}^{8, X Y}$ & $\bar{c} u \bar{u} u, \bar{u} u \bar{c} u$ & $.35 \quad .44$ & $5.1-4.5 \mathrm{TeV}$ \\
\hline $\mathcal{O}^{1, X X}$ & $\bar{c} u \bar{u} u$ & $.09 \quad .11$ & $10-9.0 \mathrm{TeV}$ \\
\hline $\mathcal{O}^{1, X Y}$ & $\bar{c} u \bar{u} u, \bar{u} u \bar{c} u$ & $.16 \quad .21$ & $7.5-6.5 \mathrm{TeV}$ \\
\hline $\mathcal{O}^{1, X X}$ & $\bar{q} d \bar{d} d$ & $.30 \quad .37$ & $5.5-4.9 \mathrm{TeV}$ \\
\hline $\mathcal{O}^{1, X Y}$ & $\bar{q} d \bar{d} d, \bar{d} d \bar{q} d$ & $\begin{array}{ll}.59 & .71\end{array}$ & $3.9-3.6 \mathrm{TeV}$ \\
\hline
\end{tabular}

Table 6. Estimated bounds on the contact interaction scale from figure 4, for the interactions of the first column with flavour structure $i j \rightarrow m n, i j=u u$ or $d d$, and $\Delta F=1$. In this table, $q=b, s$. In the third column are given the bounds on $\hat{s} / \Lambda^{2}$ from requiring $|\epsilon|<1 / 3$ or $|\epsilon|<1 / 2$. The bound in the last column is obtained with $\hat{s}=M_{\text {dijet }}^{2}=(3 \mathrm{TeV})^{2}$.

\begin{tabular}{|l|c|cc|c|}
\hline Operator & flavours minj & $\hat{s} / \Lambda^{2}$ & $\Lambda<$ \\
\hline $\mathcal{O}^{8, X X}$ & $\bar{c} u \bar{q} d$ & .36 & .43 & $5.0-4.6 \mathrm{TeV}$ \\
$\mathcal{O}^{8, X Y}$ & $\bar{c} u \bar{q} d, \bar{q} d \bar{c} u$ & .67 & .84 & $3.7-3.3 \mathrm{TeV}$ \\
$\mathcal{O}^{1, X X}, \mathcal{O}^{1, C C}$ & $\bar{c} u \bar{q} d$ & .20 & .25 & $6.7-6.0 \mathrm{TeV}$ \\
$\mathcal{O}^{1, X Y}$ & $\bar{c} u \bar{q} d, \bar{q} d \bar{c} u$ & .32 & .40 & $5.3-4.7 \mathrm{TeV}$ \\
$\mathcal{O}^{S 1}$ & $-\bar{c} u \bar{q} d+\bar{q} u \bar{c} d$ & .56 & .67 & $4.0-3.7 \mathrm{TeV}$ \\
\hline
\end{tabular}

Table 7. Estimated bounds on the contact interaction scale from figure 4, for the interactions of the first column with flavour structure $i j \rightarrow m n, i j=u d$, and $\Delta F=2$. In this table, $q=b, s$. In the third column are given the bounds on $\hat{s} / \Lambda^{2}$ from requiring $|\epsilon|<1 / 3$ or $|\epsilon|<1 / 2$. The bound in the last column is obtained with $\hat{s}=M_{\text {dijet }}^{2}=(3 \mathrm{TeV})^{2}$.

\begin{tabular}{|c|c|c|c|}
\hline Operator & flavours minj & $\hat{s} / \Lambda^{2}$ & $\Lambda<$ \\
\hline $\mathcal{O}^{8, X X}$ & $\bar{c} u \bar{d} d, \bar{u} u \bar{q} d$ & $\begin{array}{ll}.36 & .43\end{array}$ & $5.0-4.6 \mathrm{TeV}$ \\
\hline \multirow[t]{2}{*}{$\mathcal{O}^{8, X Y}$} & $\bar{c} u \bar{d} d, \bar{d} d \bar{c} u$ & & \\
\hline & $\bar{u} u \bar{q} d, \bar{q} d \bar{u} u$ & $.66 \quad .84$ & $3.7-3.3 \mathrm{TeV}$ \\
\hline $\mathcal{O}^{1, X X}, \mathcal{O}^{1, C C}$ & $\bar{u} u \bar{q} d, \bar{c} u \bar{d} d$ & $.20 \quad .25$ & $6.7-6.0 \mathrm{TeV}$ \\
\hline $\mathcal{O}^{1, X Y}$ & $\bar{c} u \bar{d} d, \bar{d} d \bar{c} u$ & & \\
\hline \multirow{3}{*}{$\mathcal{O}^{S 1}$} & $\bar{u} u \bar{q} d, \bar{q} d \bar{u} u$ & $.32 \quad .40$ & $5.3-4.7 \mathrm{TeV}$ \\
\hline & $-\bar{c} u \bar{d} d+\bar{d} u \bar{c} d$ & & \\
\hline & $-\bar{u} u \bar{q} d+\bar{q} u \bar{u} d$ & $.56 \quad .67$ & $4.0-3.7 \mathrm{TeV}$ \\
\hline
\end{tabular}

Table 8. Estimated bounds on the contact interaction scale from figure 4, for the interactions of the first column with flavour structure $i j \rightarrow m n, i j=u d$, and $\Delta F=1 . q=b$ or $s$. In the third column are given the bounds on $\hat{s} / \Lambda^{2}$ from requiring $\epsilon<1 / 3$ or $\epsilon<1 / 2$. The bound in the last column is obtained with $\hat{s}=M_{\text {dijet }}^{2}=(3 \mathrm{TeV})^{2}$. 


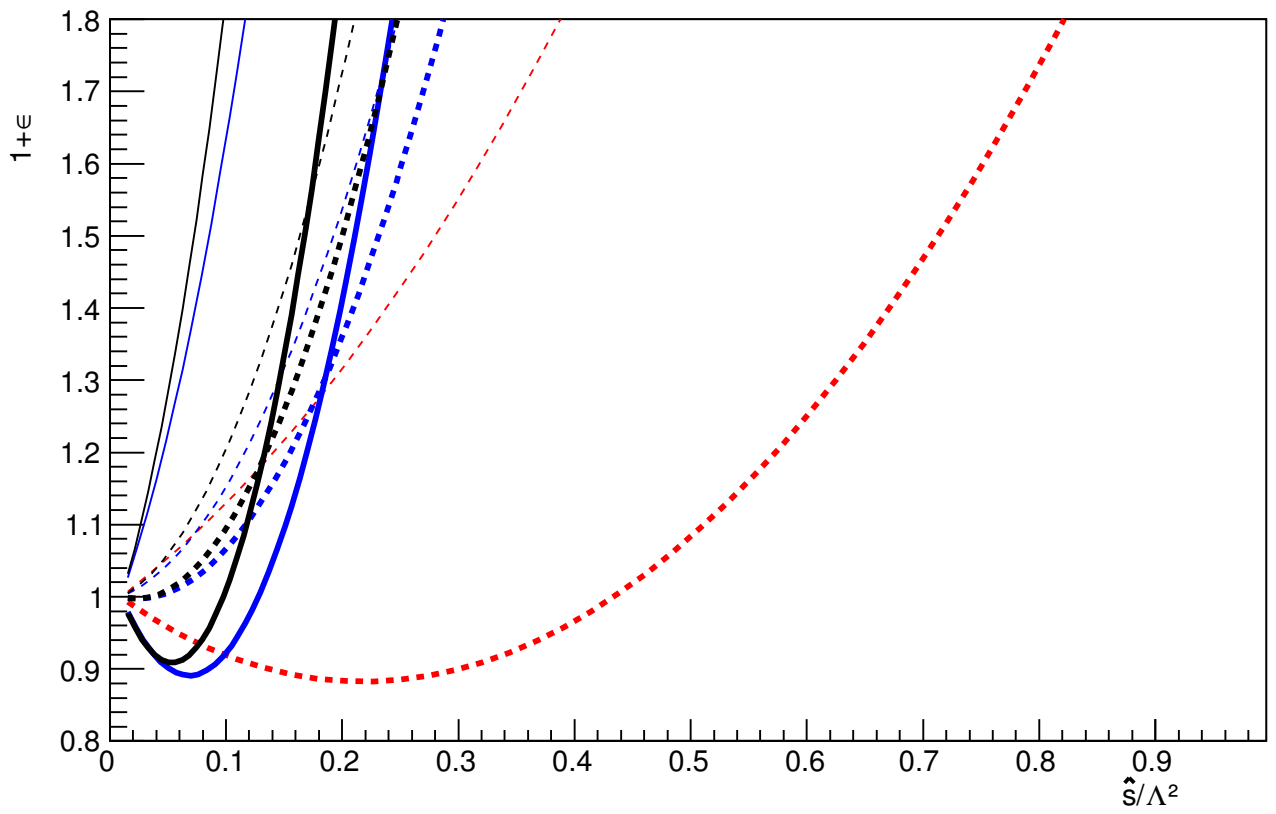

dd->dd

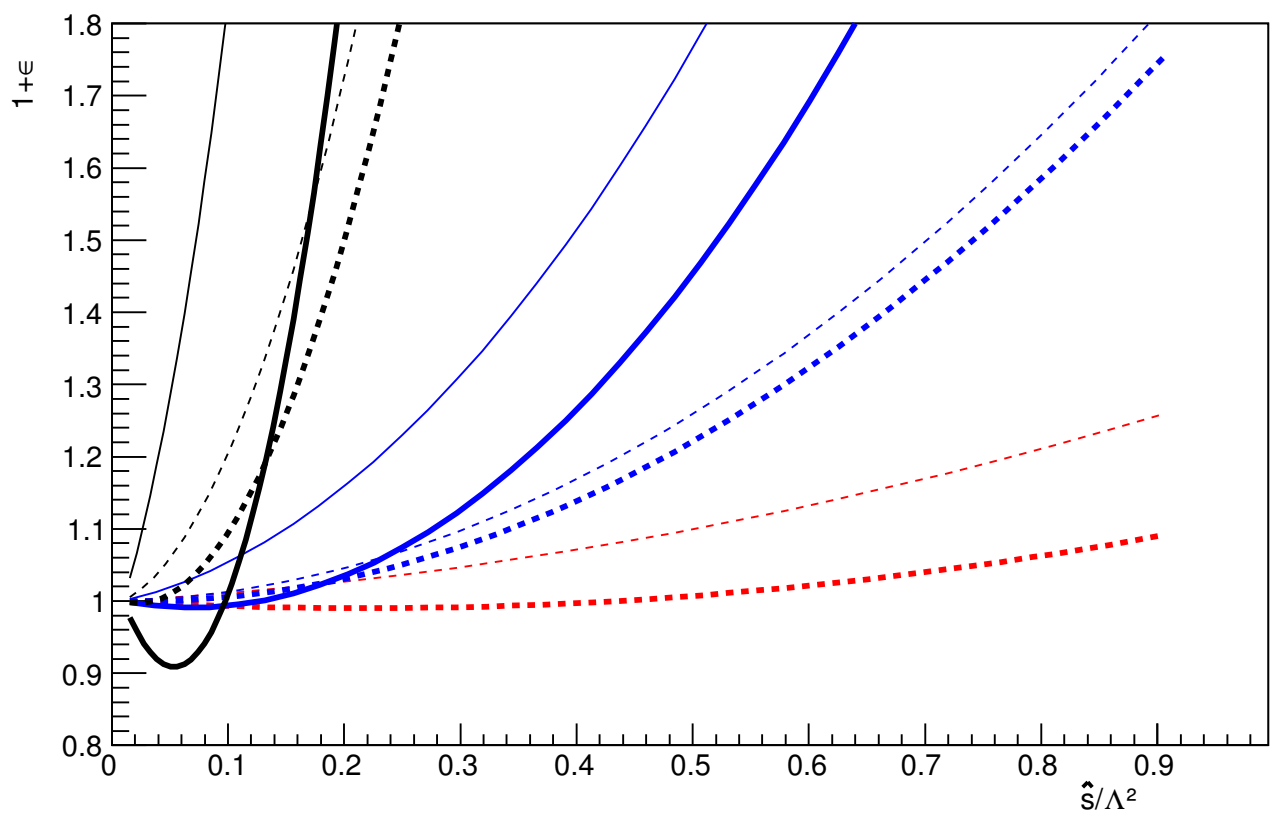

Figure 2. Ratio $1+\epsilon$ (see eq. (5.6)) as a function of $\hat{s} / \Lambda^{2}$ for $u u \rightarrow u u$ (up) and $d d \rightarrow d d$ (down) contact interactions. Continuous (dashed) lines correspond to interactions with the same (opposite) chiral projector in the two currents, and thick (thin) lines are for a positive (negative) coefficient in the Lagrangian. The lines for $\mathcal{O}^{8}$ are red, blue is $\mathcal{O}^{1}$, and Black are the flavour-summed $\mathcal{O}^{\text {pythia }}$ for comparison (The clockwise colour order for $u u \rightarrow u u$ at the origin is Black,blue,B,b,r,B,b,r,B,b. For $d d \rightarrow d d$ at the border, it is $: \mathrm{B}, \mathrm{B}, \mathrm{B}, \mathrm{B}, \mathrm{b}, \mathrm{b}, \mathrm{b}, \mathrm{b}, \mathrm{r}, \mathrm{r})$. The bounds derived on $\Lambda$ are obtained by requesting that $\hat{s} / \Lambda^{2}$ should be small enough to give $1+\epsilon \lesssim 1.33 \rightarrow 1.5$. 


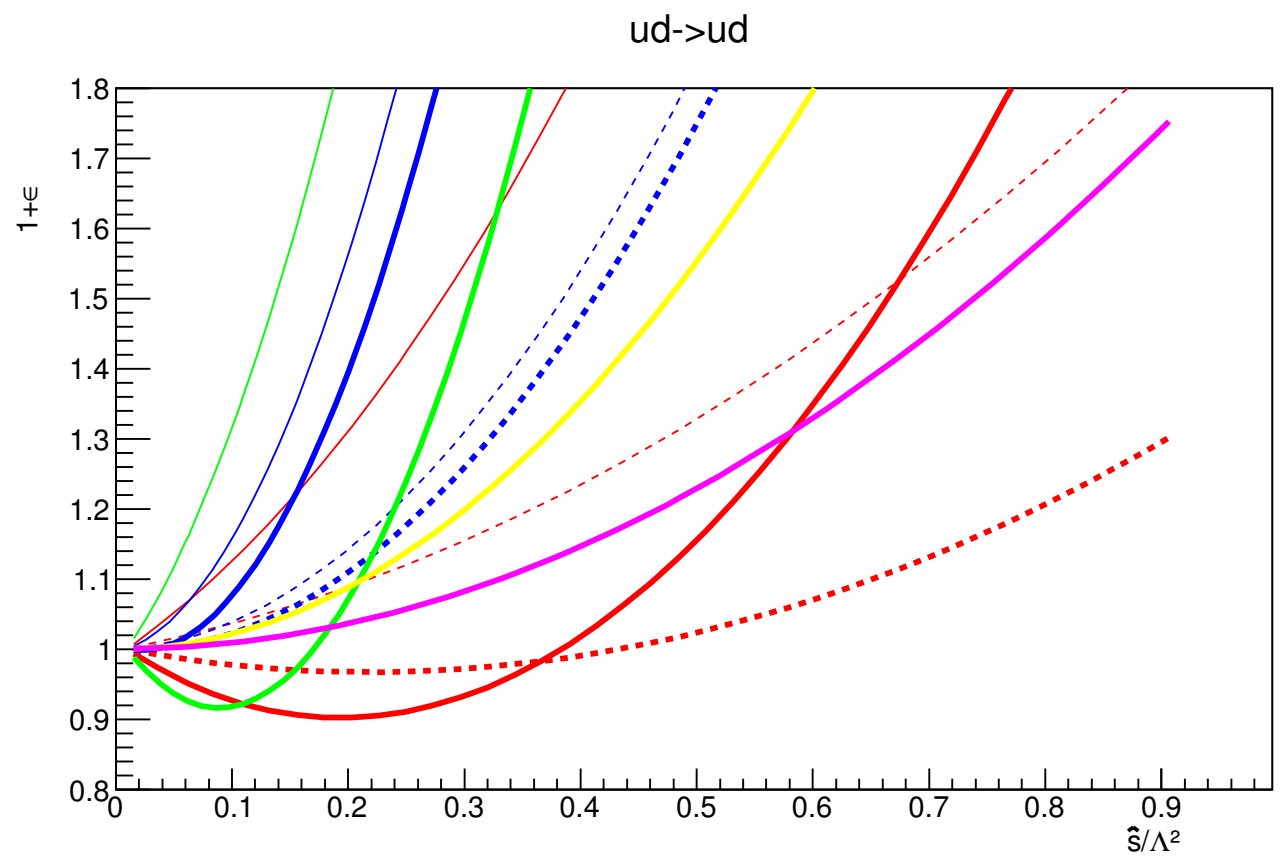

Figure 3. Ratio $1+\epsilon$ (see eq. (5.6)) as a function of $\hat{s} / \Lambda^{2}$ for $u d \rightarrow u d$ contact interactions. Continuous (dashed) lines correspond to interactions with the same (opposite) chiral projector in the two currents, and thick (thin) lines are for a positive (negative) coefficient in the Lagrangian. Red lines are for $\mathcal{O}^{8}$, blue for $\mathcal{O}^{1}$, green for $\mathcal{O}^{1, C C}$, yellow for $\mathcal{O}^{S 1}$, pink for $\mathcal{O}^{S 8}$ (The colour order clockwise at the border is $\mathrm{g}, \mathrm{b}, \mathrm{b}, \mathrm{g}, \mathrm{r}, \mathrm{b}, \mathrm{b}, \mathrm{y}, \mathrm{r}, \mathrm{r}, \mathrm{p}, \mathrm{r})$.

2. suppose the last bin in dijet mass will always have an experimental uncertainty of 20\%-30\% and remain compatible with the QCD prediction. This may be reasonable, because the bound on contact interactions profits more from going to a larger $\hat{s}$ than from reducing the statistical uncertainties to the size of the systematics. So $\epsilon$ would remain approximately $1 / 3$ (or $1 / 2$ ).

Then the estimated bounds we quote on $\hat{s} / \Lambda^{2}$ remain valid, and the bound on $\Lambda$ will be multiplied by a factor $M_{\text {dijet }}^{(\text {new) }} /(3 \mathrm{TeV})$, where $M_{\text {dijet }}^{(\text {new })}$ is the lower bound on the dijet mass of the highest bin of a future analysis.

In relation with this issue, we come back to the interpretation of the current result of CMS in the lowest- $\chi$ bin for $M_{\text {dijet }}>3 \mathrm{TeV}$ [4]. Up to now, we have considered that the spread of data below and above the QCD predictions precluded explaining with contact interactions the $\sim 30 \%$ deficit in the $1 \leq \chi \leq 3$ bin. Let us however entertain this possibility, which requires a negative contribution coming from a negative interference between QCD and contact interactions. As already discussed earlier and explicitly seen from figures 2, 3 and 4, and in the appendix, the sign and size of the interference depend on the flavour considered. It turns out that one can reduce the number of events by around $10 \%$ in the case of $O_{\text {uuuu }}^{1, X X}, O_{\text {uddu }}^{1, C C}, O_{\text {uuuu }}^{8, X Y}$ and $O_{\text {uudd }}^{8, X X}$ (with a scale of around $11 \mathrm{TeV}$ for the singlet operators and a scale around $7 \mathrm{TeV}$ for the octet operators). These operators show a similar behaviour once extrapolated to higher $M_{\text {dijet }}$. They yield a deficit of events around 
uU->cc

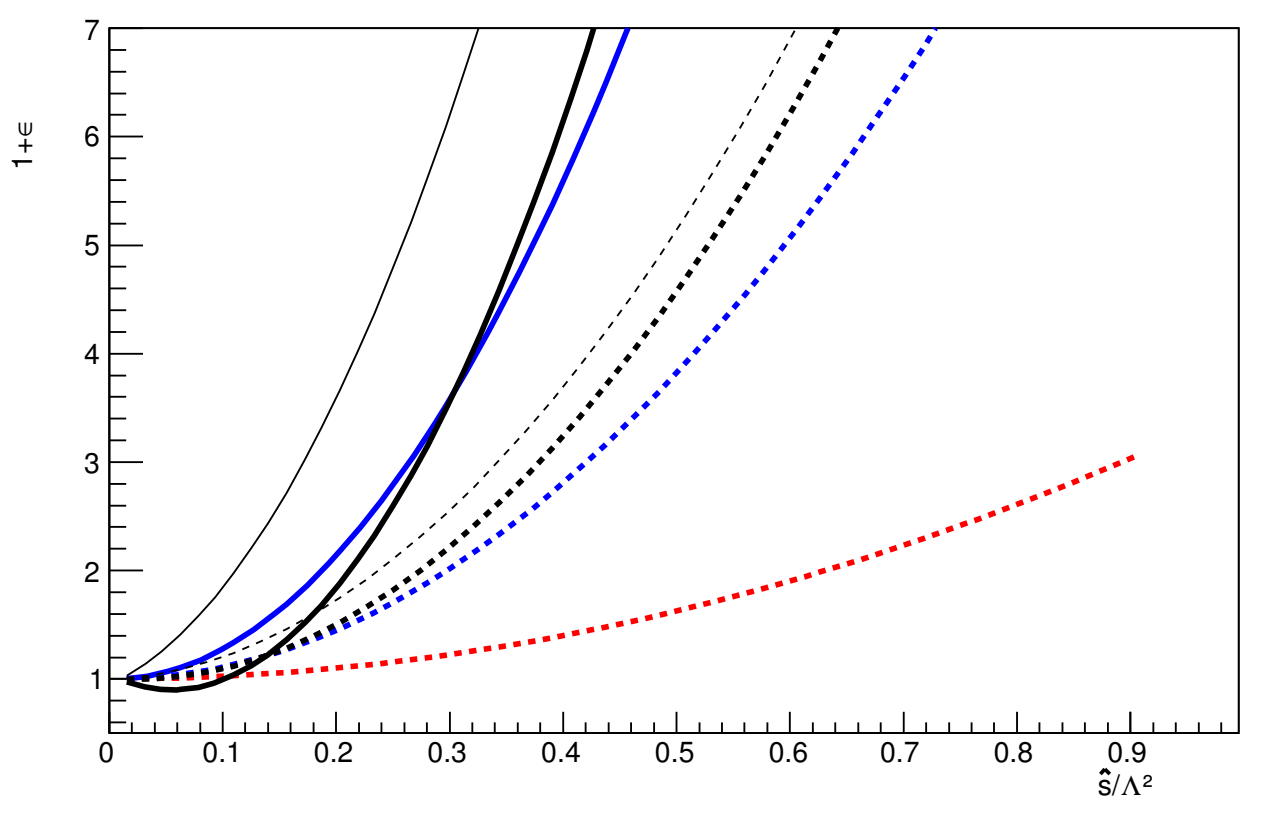

ud->cs

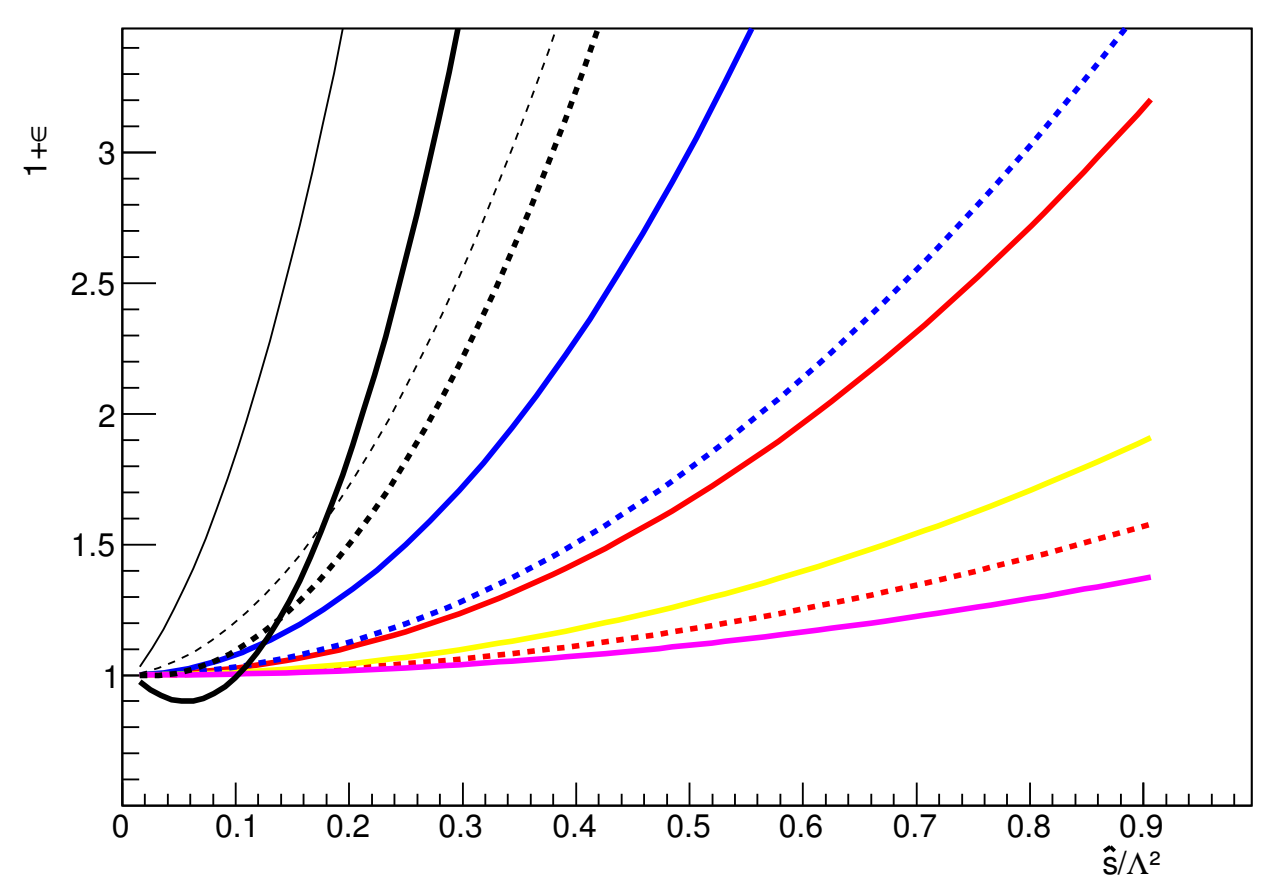

Figure 4. Ratio $1+\epsilon$ (see eq. (5.6)) as a function of $\hat{s} / \Lambda^{2}$ for flavour changing contact interactions $u u \rightarrow c c$ (up) or $u d \rightarrow c s$ (down). Continuous (dashed) lines correspond to interactions with the same (opposite) chiral projector in the two currents. There is no interference with QCD, so the sign of the contact interactions does not matter. Black are the flavour-summed $\mathcal{O}^{\text {pythia }}$ (for comparaison), red are for $\mathcal{O}^{8}$, blue for $\mathcal{O}^{1}$, yellow for $\mathcal{O}^{S 1}$, and pink for $\mathcal{O}^{S 8}$ (The colour order at the border is, for $u u \rightarrow c c:$ Black,B,blue,B,B,b,r, and for $u d \rightarrow c s: \mathrm{B}, \mathrm{B}, \mathrm{B}, \mathrm{B}, \mathrm{b}, \mathrm{b}, \mathrm{r}, \mathrm{y}, \mathrm{r}, \mathrm{p})$. 
$10 \%$ in the bin $2.4-3 \mathrm{TeV}$ (where CMS data are in very good agreement with QCD), and of around $7 \%$ in the bin $1.9-2.4 \mathrm{TeV}$. The effect is within QCD uncertainties at lower $M_{\text {dijet }}$.

At higher $M_{\text {dijet }}$, of interest in the context of the LHC upgraded to $14 \mathrm{TeV}$, the contribution from contact interactions-squared becomes larger and starts dominating over the interference with QCD: for $4 \mathrm{TeV}$, the total contribution from contact interactions approximately vanishes, and becomes positive at higher dijet mass, with an excess of $30 \%$ at $5 \mathrm{TeV}$, and more than $100 \%$ at $6 \mathrm{TeV}$. In this particular scenario, the slight deficit currently observed in the $M_{\text {dijet }}>3 \mathrm{TeV}$ bin is not enough to draw definite conclusions on the presence or absence of contact interactions, and this situation will still hold with the increase of the dijet energy, up to the point where one reaches so high dijet masses that the (now positive) contribution from contact interactions yields a significant, non ambiguous, sign of their presence.

On the other hand, going to such high dijet masses, one could in principle discriminate between singlet and octet cases, since the latter case corresponds to a scale $\Lambda \sim 6 \mathrm{TeV}$ and should thus be resolved in terms of intermediate massive states, whereas the former, linked with a scale $\Lambda \sim 11 \mathrm{TeV}$, could still remain a contact interaction if studied by a LHC running at $14 \mathrm{TeV}$.

\section{Discussion}

Contact interactions can be induced by the exchange of new resonances which are not resolved as mass eigenstates, because they are either broad or heavier than the exchanged four-momentum. For instance, the tree-level exchange of an off-shell boson of mass $M_{\text {new }} \gtrsim$ $M_{\text {dijet }}$, interacting with the quarks via a coupling $g^{\prime}$, would give $4 \pi / \Lambda^{2} \sim g^{\prime 2} / M_{\text {new }}^{2}$. As discussed in section 3.3, the dijet spectrum at hadron colliders could be sensitive to contact interactions with $4 \pi / \Lambda^{2} \gtrsim g_{s}^{2} / M_{\text {dijet }}^{2}$, provided that $M_{\text {new }}^{2} \gtrsim M_{\text {dijet }}^{2}$. So colliders can seach for contact interactions mediated by heavy particles with $\mathcal{O}(1)$ couplings to quarks.

Heavy particles interacting with quarks can evade LHC bounds on contact interactions in various ways. For instance, if new particles have a conserved parity (as is convenient to obtain dark matter), they can generate contact interactions via a closed loop of heavy new particles, with a coefficient

$$
4 \pi / \Lambda^{2} \sim g^{\prime 4} /\left(16 \pi^{2} M_{\text {new }}^{2}\right) .
$$

For $M_{\text {new }}^{2} \gtrsim M_{\text {dijet }}^{2}$, this is unobservably small at the LHC (unless $g^{\prime 2} \sim 4 \pi$ ), whereas for $M_{\text {new }}^{2}<M_{\text {dijet }}^{2}$, the new particles could be produced in pairs. Heavy new bosons that are less strongly coupled to quarks can also evade contact interaction bounds. For instance, a $Z^{\prime}$ with Standard Model couplings induces various four-quark contact interactions with a coefficient $4 \pi / \Lambda^{2} \sim g^{2} /\left(8 c_{W}^{2} M_{Z^{\prime}}^{2}\right) \ll g_{s}^{2} / M_{\text {dijet }}^{2}$, for $M_{Z^{\prime}}^{2} \gtrsim M_{\text {dijet }}^{2}$.

It is interesting to compare the collider bounds on four-quark contact interactions to limits from precision flavour physics, despite that a contact interaction could be present at lower energies while absent at $3 \mathrm{TeV}$. The first step should be to evolve the operator coefficients between the $\mathrm{TeV}$ scale and low energy (e.g. $\left.m_{b}\right)$ :

$$
\left.\left.\left.\frac{4 \pi}{\Lambda^{2}}\right|_{m_{b}} \simeq c \frac{4 \pi}{\Lambda^{2}}\right|_{3 \mathrm{TeV}} \sim\left(\frac{\alpha_{s}(3 \mathrm{TeV})}{\alpha_{s}\left(m_{b}\right)}\right)^{\gamma / 2 \beta_{0}} \frac{4 \pi}{\Lambda^{2}}\right|_{3 \mathrm{TeV}}
$$


where $\beta_{0}=\left(11 N_{c}-2 N_{f}\right) / 12 \pi$. For a few cases where we know the anomalous dimension $\gamma$ of the contact interaction, $0.5<c<2[25,26]$, so we neglect the running and use at low energy the bound on $4 \pi / \Lambda^{2}$ obtained at $3 \mathrm{TeV}$. Due to the importance of Fermi interaction in low-energy precision physics, it is convenient to define the parameter $\beta$ as

$$
\frac{4 \pi}{\Lambda^{2}}=\beta \frac{4 G_{F}}{\sqrt{2}} \Rightarrow \beta=\frac{4 \pi v^{2}}{\Lambda^{2}}=\left(\frac{0.6 \mathrm{TeV}}{\Lambda}\right)^{2}
$$

so the collider bounds discussed previously, ranging from 3 to $11 \mathrm{TeV}$, imply $\beta \lesssim 4 \times 10^{-2} \rightarrow$ $3 \times 10^{-3}$. Let us add that flavour physics and collider searches do not have the same scope in probing the flavour structure of contact interactions. As discussed above, proton-proton collider searches have the potential to search four-quark contact interactions involving at least two quarks of the first generation, but the two other quark lines are left free (and perhaps could be identified through jet tagging). In flavour physics, neutral-meson mixing is a sensitive probe of flavour off-diagonal $(\Delta F=2)$ contact interactions [26-28], with particularly stringent bounds on $\Lambda$ for operators inducing kaon mixing (assuming, as we have done here, that the coefficients $\eta$ are $O(1)$ ). Bounds on other four-quark operators could in principle come from processes where a meson decays into two lighter mesons nonleptonically - however, such processes are very challenging from the theoretical point of view, and can hardly be considered as useful to set constraints on contact interactions.

If a signal for contact interactions was observed at the LHC, it could indicate strongly coupled New Physics at a scale just beyond the reach of the LHC, or the (perturbative) exchange of a (broad) resonance in $t$ or $s$-channel. In either case, it would be interesting to identify the flavour of the final state jets - not only to distinguish gluons and heavy flavours $(b, c)$ from light quarks, but even to distinguish $u$ from $d$ jets using jet charge. This would allow one to predict the expected rate for the crossed process. For instance, if a $t$-channel $Z^{\prime}$ mediates $u u \rightarrow u u$, then it could also be searched for as a bump in $u \bar{u} \rightarrow u \bar{u}$ whereas an $s$-channel diquark inducing the contact interactions $u d \rightarrow c s$ is not worth searching for in $t$ channel.

\section{Summary}

Many models with new particles at high energy have contact interactions as their lowenergy footprints. As such, contact interactions are a parametrisation of New Physics, so it is important that constraints on upon them be as widely applicable as possible. Current collider bounds are calculated for a palette of colour-singlet interactions (no colour matrices in the vertex), summed on flavour. In this paper, we estimate bounds on an almost complete basis of four-quark contact interactions, with specified flavour indices mediating $q_{i} q_{j} \rightarrow q_{m} q_{n}$, where $q_{i}, q_{j}$ are first-generation quarks.

We start from a basis of dimension six, Standard Model gauge-invariant, four-quark operators. After electroweak symmetry breaking, each operator induces one or several fourquark interactions. We constrain the coefficients of these effective interactions by turning them on one at a time. We prefer to constrain the coefficients of the effective interactions, rather than the gauge-invariant operators, because this allows us to apply the bounds 
to a different operator basis. The effective interactions (almost) do not interfere among themselves, so that the bounds obtained by turning them on one at a time are conservative. A more stringent bound could apply in the presence of several contact interactions.

The bounds profit from a convenient choice of variables made by the experimental collaborations, which allows one to approximate the $p p \rightarrow$ dijets cross section as an integral over parton distribution functions multiplying a partonic cross section. We estimate the expected limit on contact interactions by comparing their partonic cross sections to the leading order QCD prediction. The data agree with $\mathrm{QCD}$, so we require that the contact interactions contribute less than $1 / 3$ or $1 / 2$ of the QCD expectation. Our bounds are listed in tables 3-8. Our flavoured estimates are generically lower than the limits of ATLAS and CMS, who constrain a flavour-sum of contact interactions given in eq. (4.1). Our estimate for this flavour-summed operator is comparable to the experimental bounds.

The original aim of this paper was to identify "classes" among the large collection of contact interactions, such that a bound obtained on a representative of the class could be translated by some simple rule to the others. The situation is however more complicated: the obstacle seems to be the interference between QCD and the contact interaction, which precludes any simple scaling of the limit on $\hat{s} / \Lambda^{2}$ from one interaction to another. ${ }^{5}$ An interesting feature of our analytic recipe is that it constrains the dimensionless ratio $M_{\text {dijet }}^{2} / \Lambda^{2}$ for each contact interaction. The future LHC, with more energy and luminosity, will be able to probe a higher dijet mass than the $M_{\text {dijet }}=3 \mathrm{TeV}$ [4] used here to extract bounds on $\Lambda$. The estimated bounds on $\Lambda$ from tables 3-8 should therefore scale as $M_{\text {dijet }}($ new $) /(3 \mathrm{TeV})$, under assumptions given in section 5.3.

Finally, we discussed briefly which contact interactions could explain a moderate deficit of events in the current data for the $1 \leq \chi \leq 3$ and $M_{\text {dijet }}>3 \mathrm{TeV}$ bin, and how this would extrapolate to higher dijet masses. It will be particular interesting to see how the spread of results obtained by the experimental collaborations evolve as luminosity and energy increase.

\section{Acknowledgments}

We thank M. Gouzevitch, A. Hinzmann, G. Salam, and P. Skands for useful conversations. This project was performed in the context of the Lyon Institute of Origins, ANR-10-LABX-66.

\section{A Kinematics and cross sections}

This appendix gives the contact interaction correction, in sections labeled by flavour structure, for a larger set of contact interactions than the basis of section 3.2 (in particular, we give cross sections for octet interactions involving four $u$ - or $d$-type quarks, although these are not in the list of section 3.2). The formulae are labeled to the right by the contact inter-

\footnotetext{
${ }^{5}$ It is mildly surprising that a limit on contact interactions at a hadron collider can be estimated analytically; however, in practice, it may be just as simple to use Madgraph5 [29].
} 
action to which they apply. The partonic cross sections mediated by contact interactions are given in several references $[22,30],{ }^{6}$ and are collected below for convenience.

\section{A.1 Definitions}

For momenta as given in the first diagram of figure 1, with time running left to right, the Mandelstam variables are defined as

$$
\begin{aligned}
& \hat{s}=\left(k_{1}+k_{2}\right)^{2}=2 k_{1} \cdot k_{2}=2 p_{1} \cdot p_{2}=4 E^{* 2} \\
& \hat{t}=\left(k_{1}-p_{1}\right)^{2}=-2 k_{1} \cdot p_{1}=-2 p_{2} \cdot k_{2}=-2 E^{* 2}\left(1-\cos \theta^{*}\right) \\
& \hat{u}=\left(k_{1}-p_{2}\right)^{2}=-2 k_{1} \cdot p_{2}=-2 p_{1} \cdot k_{2}=-2 E^{* 2}\left(1+\cos \theta^{*}\right)
\end{aligned}
$$

where the last expressions are in the parton centre-of mass frame. If time runs upwards in the same diagram, it describes $q \bar{q}$ annihilations, and the Mandelstam variables exchange their definitions:

$$
\hat{s}_{\text {scat }}=\hat{u}_{\text {ann }}, \quad \hat{t}_{\text {scat }}=\hat{s}_{\text {ann }}, \quad \hat{u}_{\text {scat }}=\hat{t}_{\text {ann }} .
$$

In the following, we give various partonic differential cross sections,

$$
\frac{d \hat{\sigma}}{d \hat{t}}=\frac{\overline{|\mathcal{M}|}^{2}}{16 \pi \hat{s}^{2}} \longrightarrow \frac{d \hat{\sigma}}{d \chi}=\left.\frac{\hat{t}^{2}}{\hat{s}}\left(\frac{d \hat{\sigma}}{d \hat{t}}(\hat{s}, \hat{t}, \hat{u})+\frac{d \hat{\sigma}}{d \hat{t}}(\hat{s}, \hat{u}, \hat{t})\right)\right|_{\theta^{*}<\pi / 2}
$$

where $\overline{\mid \mathcal{M}}^{2}$ is averaged over incoming spins and colours, and contains a factor $1 / 2$ when the final state particles are identical. Since the change of variables to $\chi$ is simple for $\cos \theta^{*}>0$, we restrict $0 \leq \theta^{*} \leq \pi / 2$, and add $d \hat{\sigma} / d \hat{t}$ with $\hat{t} \leftrightarrow \hat{u}$.

We include the QCD contribution, the contact-interaction interference with QCD (and sometimes QED), and the contact interaction squared. We neglect the pure QED contribution (subdominant with respect to QCD), but include QED-contact interference when there is no QCD-contact interference. Indeed the interference term $\propto \alpha_{e m} \hat{s} / \Lambda^{2}$ can reduce the cross section and have a minor effect on the bound.

\section{A.2 $q_{i} g \rightarrow q_{i} g-$ QCD only}

$$
\frac{d \sigma}{d \hat{t}}=\frac{4 \pi \alpha_{s}^{2}}{9 \hat{s}^{2}}\left[-\frac{\hat{u}}{\hat{s}}-\frac{\hat{s}}{\hat{u}}+\frac{9}{4} \frac{\hat{s}^{2}+\hat{u}^{2}}{\hat{t}^{2}}\right]
$$

\section{A.3 $q_{i} q_{j} \rightarrow q_{m} q_{n}$}

$$
\text { A.3.1 } q q \rightarrow q q,(i=j=m=n)
$$

$$
\frac{d \hat{\sigma}}{d \hat{t}}(q q \rightarrow q q)=\frac{2 \pi \alpha_{s}^{2}}{9 \hat{s}^{2}}\left[\frac{\hat{s}^{2}+\hat{u}^{2}}{\hat{t}^{2}}+\frac{\hat{t}^{2}+\hat{s}^{2}}{\hat{u}^{2}}-\frac{2}{3} \frac{\hat{s}^{2}}{\hat{t} \hat{u}}\right] \equiv Q C D
$$

\footnotetext{
${ }^{6}$ These formulae do not always agree with ref. [31], and disagree on the sign of some interferences with respect to ref. [32].
} 


$$
\begin{aligned}
\frac{d \hat{\sigma}}{d \hat{t}}(q q \rightarrow q q) & =Q C D-\frac{\eta_{8, X X}}{\Lambda^{2}} \frac{4 \pi \alpha_{s}}{27} \frac{\hat{s}}{\hat{u} \hat{t}}+\frac{\left|\eta_{8, X X}\right|^{2}}{\Lambda^{4}} \frac{4 \pi}{27} & \mathcal{O}^{8, R R}, \mathcal{O}^{8, L L} \\
& =Q C D+\frac{\eta_{8, X Y}}{\Lambda^{2}} \frac{4 \pi \alpha_{s}}{9 \hat{s}^{2}} \frac{\hat{u}^{3}+\hat{t}^{3}}{\hat{t} \hat{u}}+\frac{\left|\eta_{8, X Y}\right|^{2}}{\Lambda^{4}} \frac{2 \pi}{9} \frac{\left(\hat{u}^{2}+\hat{t}^{2}\right)}{\hat{s}^{2}} & \mathcal{O}^{8, R L}=\mathcal{O}^{8, R L} \\
& =Q C D-\frac{\eta_{1, X X}}{\Lambda^{2}} \frac{4 \pi \alpha_{s}}{9} \frac{\hat{s}}{\hat{u} \hat{t}}+\frac{\left|\eta_{1, X X}\right|^{2}}{\Lambda^{4}} \frac{4 \pi}{3} & \mathcal{O}^{1, L L}, \mathcal{O}^{1, R R} \\
& =Q C D+\eta_{1, X Y} \frac{2 \pi \alpha_{e m}}{\Lambda^{2}}\left(\frac{\hat{u}^{2}}{\hat{t}}+\frac{\hat{t}^{2}}{\hat{u}}\right) \frac{1}{\hat{s}^{2}}+\frac{\left|\eta_{1, X Y}\right|^{2}}{\Lambda^{4}} \pi \frac{\left(\hat{t}^{2}+\hat{u}^{2}\right)}{\hat{s}^{2}} & \mathcal{O}^{1, R L}=\mathcal{O}^{1, R L} .
\end{aligned}
$$

A factor of $1 / 2$ for identical fermions in the final state is included in eqs. (A.4), (A.5) and (A.7), When the final state quarks have opposite chirality, they are not identical, but the operators $\mathcal{O}^{R L}=\mathcal{O}^{, R L}$ are, so its the same to include one operator for distinct fermions, or two operators for identical fermions.

Interference with QED is included for the singlet operators involving quarks of different chirality: $\mathcal{O}^{1, R L}+\mathcal{O}^{1, R L}$. The interference with QCD is absent because the spinor traces vanish: a non-zero trace must contain an even number of colour matrices, so both the QCD vertices must appear in the trace. Therefore there is only one trace, in which appear both contact vertices, with conflicting chiral projection operators. However, QED has no colour matrices, so there is an interference term with two spinor traces.

\section{A.3.2 $q q^{\prime} \rightarrow q q^{\prime}, i=m \neq j=n$}

$$
\begin{array}{rll}
\frac{d \hat{\sigma}}{d \hat{t}}(u d \rightarrow u d)= & \frac{4 \pi \alpha_{s}^{2}}{9 \hat{t}^{2}} \frac{\hat{s}^{2}+\hat{u}^{2}}{\hat{s}^{2}} \equiv Q C D^{\prime} \\
= & Q C D^{\prime}+\frac{\eta_{8, X X}}{\Lambda^{2}} \frac{4 \pi \alpha_{s}}{9 \hat{t}}+\frac{\left|\eta_{8, X X}\right|^{2}}{\Lambda^{4}} \frac{2 \pi}{9} & \mathcal{O}^{8, X X} \\
= & Q C D^{\prime}+\frac{\eta_{8, X Y}}{\Lambda^{2}} \frac{4 \pi \alpha_{s}}{9} \frac{\hat{u}^{2}}{\hat{t} \hat{s}^{2}}+\frac{\left|\eta_{8, X Y}\right|^{2}}{\Lambda^{4}} \frac{2 \pi}{9} \frac{\hat{u}^{2}}{\hat{s}^{2}} & \mathcal{O}^{8, X Y} \\
= & Q C D^{\prime}+2 \pi \eta_{1, X X} \frac{\alpha_{e m}}{\Lambda^{2}} \frac{1}{\hat{t}}+\pi \frac{\left|\eta_{1, X X}\right|^{2}}{\Lambda^{4}} & \mathcal{O}^{1, R R} \\
= & Q C D^{\prime}+2 \pi \eta_{1, L L} \frac{\alpha_{e m}}{\Lambda^{2}} \frac{1}{\hat{t}}+\frac{8 \pi}{9} \eta_{1, C C} \frac{\alpha}{\Lambda^{2}} \frac{1}{\hat{t}} & \\
& +\pi\left(\frac{\left|\eta_{1, L L}\right|^{2}}{\Lambda^{4}}+\frac{2}{3} \frac{\eta_{1, C C} \eta_{1, L L}}{\Lambda^{2} \Lambda^{2}}+\frac{\left|\eta_{1, C C}\right|^{2}}{\Lambda^{4}}\right) & \mathcal{O}^{1, L L}+\mathcal{O}^{1, C C} \\
= & Q C D^{\prime}+2 \pi \eta_{1, X Y} \frac{\alpha_{e m}}{\Lambda^{2}} \frac{\hat{u}^{2}}{\hat{t}^{2}}+\pi \frac{\left|\eta_{1, X Y}\right|^{2}}{\Lambda^{4}} \frac{\hat{u}^{2}}{\hat{s}^{2}} & \mathcal{O}^{1, X Y} \\
= & Q C D^{\prime}+\frac{\pi}{6 \Lambda^{4}} \frac{4 \hat{u}^{2}+4 \hat{t}^{2}-\hat{s}^{2}}{\hat{s}^{2}} & \mathcal{O}^{S 1} \\
= & Q C D^{\prime}+\frac{\pi}{27 \Lambda^{4}} \frac{2 \hat{u}^{2}+2 \hat{t}^{2}+\hat{s}^{2}}{\hat{s}^{2}} & \mathcal{O}^{S 8}
\end{array}
$$


Notice that for $u d \rightarrow u d, \mathcal{O}^{1, L R}$ is different from $\mathcal{O}^{1, R L}$. so their contributions are not summed in the above formulae.

Interference with QED is included when there is no interference with QCD, and the interference between $\mathcal{O}^{1, L L}$ and $\mathcal{O}^{1, C C}$ is given, although we constrain the two operators separately.

\section{A.3.3 $\quad q^{\prime} q^{\prime} \rightarrow q q$}

If the quark flavour changes at the contact interaction, there is no interference with QCD. However, there are two contact interaction diagrams, and an interference term when the initial and final states contain identical quarks. At the LHC, this can describe $u u \rightarrow c c$, $d d \rightarrow s s$, and $d d \rightarrow b b$.

$$
\begin{aligned}
\frac{d \hat{\sigma}}{d \hat{t}} & =\frac{\left|\eta_{8, X X}\right|^{2}}{\Lambda^{4}} \frac{4 \pi}{27} & \mathcal{O}^{8, X X} \\
& =\frac{\left|\eta_{8, X Y}\right|^{2}}{\Lambda^{4}} \frac{2 \pi}{9} \frac{\left(\hat{t}^{2}+\hat{u}^{2}\right)}{\hat{s}^{2}} & \mathcal{O}^{8, X Y}=\mathcal{O}^{8, Y X} \\
& =\frac{\left|\eta_{1, X X}\right|^{2}}{\Lambda^{4}} \frac{4 \pi}{3} & \mathcal{O}^{1, X X} \\
& =\frac{\left|\eta_{1, X Y}\right|^{2}}{\Lambda^{4}} \pi \frac{\left(\hat{t}^{2}+\hat{u}^{2}\right)}{\hat{s}^{2}} & \mathcal{O}^{1, X Y}=\mathcal{O}^{1, Y X} .
\end{aligned}
$$

A factor of $1 / 2$ for identical fermions in the final state is included. In practice, this list is just last terms from $q q \rightarrow q q$.

\section{A.3.4 $q^{\prime} q^{\prime \prime} \rightarrow q q$, or $q q \rightarrow q^{\prime} q^{\prime \prime}$}

In the case where there are identical fermions in either the initial or final states, but not both (at the LHC: $u u \rightarrow u c, d d \rightarrow d s, d d \rightarrow d b, d d \rightarrow s b$ ), there are still two diagrams, but no interference term:

$$
\begin{aligned}
\frac{d \hat{\sigma}}{d \hat{t}}(d d \rightarrow s b) & =\frac{\left|\eta_{8, X X}\right|^{2}}{\Lambda^{4}} \frac{4 \pi}{9} & \mathcal{O}^{8, X X} \\
& =\frac{\left|\eta_{8, X Y}\right|^{2}}{\Lambda^{4}} \frac{2 \pi}{9} \frac{\left(\hat{t}^{2}+\hat{u}^{2}\right)}{\hat{s}^{2}} & \mathcal{O}^{8, X Y} \\
& =\frac{\left|\eta_{1, X X}\right|^{2}}{\Lambda^{4}} 2 \pi & \mathcal{O}^{1, X X} \\
& =\frac{\left|\eta_{1, X Y}\right|^{2}}{\Lambda^{4}} \pi \frac{\hat{t}^{2}+\hat{u}^{2}}{\hat{s}^{2}} & \mathcal{O}^{1, X Y}
\end{aligned}
$$

and in the case where the identical fermions are in the final state, the given formulae should be multiplied by $1 / 2$. 


\section{A.3.5 $q^{\prime \prime} q \rightarrow q q^{\prime}$ and any vertex with more than three different flavours}

At the LHC this can describe $u d \rightarrow c s, u d \rightarrow c b$, and also $u d \rightarrow u s, u d \rightarrow u b, u d \rightarrow c d$ :

$$
\begin{array}{rlrl}
\frac{d \hat{\sigma}}{d \hat{t}} & =\frac{\left|\eta_{8, X X}\right|^{2}}{\Lambda^{4}} \frac{2 \pi}{9}, & & \mathcal{O}^{8, X X} \\
& =\frac{\left|\eta_{8, X Y}\right|^{2}}{\Lambda^{4}} \frac{2 \pi}{9} \frac{\hat{u}^{2}}{\hat{s}^{2}}, & & \mathcal{O}^{8, X Y} \\
& =\frac{\left|\eta_{1, X X}\right|^{2}}{\Lambda^{4}} \pi, & & \mathcal{O}^{1, X X}, \quad \mathcal{O}^{1, C C} \\
& =\frac{\left|\eta_{1, X Y}\right|^{2}}{\Lambda^{4}} \pi \frac{\hat{u}^{2}}{\hat{s}^{2}}, & \mathcal{O}^{1, X Y} & \\
& =\frac{\pi}{12 \Lambda^{4}} \frac{4 \hat{u}^{2}+4 \hat{t}^{2}-\hat{s}^{2}}{\hat{s}^{2}} & \mathcal{O}^{S 1} \\
& =\frac{\pi}{54 \Lambda^{4}} \frac{2 \hat{u}^{2}+2 \hat{t}^{2}+\hat{s}^{2}}{\hat{s}^{2}} & \mathcal{O}^{S 8} .
\end{array}
$$

\section{$\mathrm{A} .4 \quad \boldsymbol{q}_{i} \overline{\boldsymbol{q}}_{m} \rightarrow \overline{\boldsymbol{q}}_{j} \boldsymbol{q}_{n}$}

For contact interactions with two incident first generation quarks, the best bounds arise from $q q \rightarrow q q$. However, a "flavour diagonal" interaction involving a quark and anti-quark of the first generation, going to a quark and anti-quark of a higher generation, is better constrained by the Tevatron, who had valence $\bar{q}_{1} q_{1}$ in the initial state. The cross sections for contact interactions in quark-anti-quark collisions, can be obtained by crossing (A.2), the previous formulae, and removing, if neccessary, the factor of $1 / 2$ for identical fermions in the final state.

Open Access. This article is distributed under the terms of the Creative Commons Attribution License (CC-BY 4.0), which permits any use, distribution and reproduction in any medium, provided the original author(s) and source are credited.

\section{References}

[1] W. Buchmüller and D. Wyler, Effective Lagrangian Analysis of New Interactions and Flavor Conservation, Nucl. Phys. B 268 (1986) 621 [INSPIRE].

[2] B. Grzadkowski, M. Iskrzynski, M. Misiak and J. Rosiek, Dimension-Six Terms in the Standard Model Lagrangian, JHEP 10 (2010) 085 [arXiv: 1008.4884] [INSPIRE].

[3] ATLAS collaboration, ATLAS search for new phenomena in dijet mass and angular distributions using pp collisions at $\sqrt{s}=7$ TeV, JHEP 01 (2013) 029 [arXiv:1210.1718] [INSPIRE].

[4] CMS collaboration, Search for quark compositeness in dijet angular distributions from pp collisions at $\sqrt{s}=7 \mathrm{TeV}$, JHEP 05 (2012) 055 [arXiv:1202.5535] [INSPIRE].

[5] CMS collaboration, Search for contact interactions using the inclusive jet $p_{T}$ spectrum in $p p$ collisions at $\sqrt{s}=7 \mathrm{TeV}$, Phys. Rev. D 87 (2013) 052017 [arXiv:1301.5023] [INSPIRE].

[6] D0 collaboration, V.M. Abazov et al., Measurement of dijet angular distributions at $\sqrt{s}=1.96 \mathrm{TeV}$ and searches for quark compositeness and extra spatial dimensions, Phys. Rev. Lett. 103 (2009) 191803 [arXiv:0906.4819] [INSPIRE]. 
[7] M. Redi, V. Sanz, M. de Vries and A. Weiler, Strong Signatures of Right-Handed Compositeness, JHEP 08 (2013) 008 [arXiv:1305.3818] [INSPIRE].

[8] R.S. Chivukula, E.H. Simmons and N. Vignaroli, A Flavorful Top-Coloron Model, Phys. Rev. D 87 (2013) 075002 [arXiv:1302.1069] [INSPIRE].

[9] Y. Bai, J.L. Hewett, J. Kaplan and T.G. Rizzo, LHC Predictions from a Tevatron Anomaly in the Top Quark Forward-Backward Asymmetry, JHEP 03 (2011) 003 [arXiv:1101.5203] [INSPIRE].

[10] U. Haisch and S. Westhoff, Massive Color-Octet Bosons: Bounds on Effects in Top-Quark Pair Production, JHEP 08 (2011) 088 [arXiv:1106. 0529] [INSPIRE].

[11] O. Domenech, A. Pomarol and J. Serra, Probing the SM with Dijets at the LHC, Phys. Rev. D 85 (2012) 074030 [arXiv:1201.6510] [INSPIRE].

[12] M. Carpentier and S. Davidson, Constraints on two-lepton, two quark operators, Eur. Phys. J. C 70 (2010) 1071 [arXiv: 1008.0280] [INSPIRE].

[13] A.E. Nelson, Contact terms, compositeness and atomic parity violation, Phys. Rev. Lett. 78 (1997) 4159 [hep-ph/9703379] [INSPIRE].

[14] J. Gao, C.S. Li, J. Wang, H.X. Zhu and C.-P. Yuan, Next-to-leading QCD effect to the quark compositeness search at the LHC, Phys. Rev. Lett. 106 (2011) 142001 [arXiv:1101.4611] [INSPIRE].

[15] E. Eichten, K.D. Lane and M.E. Peskin, New Tests for Quark and Lepton Substructure, Phys. Rev. Lett. 50 (1983) 811 [INSPIRE].

[16] T. Sjöstrand, S. Mrenna and P.Z. Skands, A Brief Introduction to PYTHIA 8.1, Comput. Phys. Commun. 178 (2008) 852 [arXiv:0710.3820] [INSPIRE].

[17] T. Sjöstrand, S. Mrenna and P.Z. Skands, PYTHIA 6.4 Physics and Manual, JHEP 05 (2006) 026 [hep-ph/0603175] [INSPIRE].

[18] H.-L. Lai et al., New parton distributions for collider physics, Phys. Rev. D 82 (2010) 074024 [arXiv: 1007.2241] [INSPIRE].

[19] L. Hartgring, E. Laenen and P. Skands, Antenna Showers with One-Loop Matrix Elements, JHEP 10 (2013) 127 [arXiv: 1303.4974] [INSPIRE].

[20] P. Skands, Talk on generator tuning Physics and Generator Tuning, CERN, October 18 (2013), http://skands.web.cern.ch/skands/slides/.

[21] Particle Data Group collaboration, J. Beringer et al., Review of Particle Physics (RPP), Phys. Rev. D 86 (2012) 010001 [INSPIRE].

[22] R.S. Chivukula, A.G. Cohen and E.H. Simmons, New strong interactions at the Tevatron?, Phys. Lett. B 380 (1996) 92 [hep-ph/9603311] [InSPIRE].

[23] H. Potter and G. Valencia, Probing lepton gluonic couplings at the LHC, Phys. Lett. B 713 (2012) 95 [arXiv: 1202.1780] [INSPIRE].

[24] L. Apanasevich, S. Upadhyay, N. Varelas, D. Whiteson and F. Yu, Sensitivity of potential future pp colliders to quark compositeness, arXiv:1307.7149 [INSPIRE].

[25] A.J. Buras, Weak Hamiltonian, CP-violation and rare decays, hep-ph/9806471 [INSPIRE].

[26] UTFIT collaboration, M. Bona et al., Model-independent constraints on $\Delta F=2$ operators and the scale of new physics, JHEP 03 (2008) 049 [arXiv:0707.0636] [INSPIRE]. 
[27] G. Isidori, Y. Nir and G. Perez, Flavor Physics Constraints for Physics Beyond the Standard Model, Ann. Rev. Nucl. Part. Sci. 60 (2010) 355 [arXiv:1002.0900] [InSPIRE].

[28] J. Charles, S. Descotes-Genon, Z. Ligeti, S. Monteil, M. Papucci and K. Trabelsi, Future sensitivity to new physics in $B_{d}, B_{s}$ and $K$ mixings, Phys. Rev. D 89 (2014) 033016 [arXiv: 1309.2293] [INSPIRE].

[29] J. Alwall, M. Herquet, F. Maltoni, O. Mattelaer and T. Stelzer, MadGraph 5: Going Beyond, JHEP 06 (2011) 128 [arXiv:1106.0522] [INSPIRE].

[30] E. Argyres et al., Alternative symmetry breaking and exotica, CERN-TH-5980-91 (1991).

[31] E. Eichten, I. Hinchliffe, K.D. Lane and C. Quigg, Super Collider Physics, Rev. Mod. Phys. 56 (1984) 579 [Addendum ibid. 58 (1986) 1065] [INSPIRE].

[32] K.D. Lane, Electroweak and flavor dynamics at hadron colliders, hep-ph/9605257 [INSPIRE]. 